\title{
A patient-safety and professional perspective on non-conveyance in ambulance care: a systematic review
}

Remco H.A. Ebben ${ }^{1 *}$, Lilian C.M. Vloet ${ }^{1,2}$, Renate F. Speijers ${ }^{1,3}$, Nico W. Tönjes ${ }^{1,4,5}$, Jorik Loef ${ }^{1}$, Thomas Pelgrim', Margreet Hoogeveen ${ }^{6}$ and Sivera A.A. Berben ${ }^{1,2,7}$

\begin{abstract}
Background: This systematic review aimed to describe non-conveyance in ambulance care from patient-safety and ambulance professional perspectives. The review specifically focussed at describing (1) ambulance non-conveyance rates, (2) characteristics of non-conveyed patients, (3) follow-up care after non-conveyance, (4) existing guidelines or protocols, and (5) influencing factors during the non-conveyance decision making process.

Methods: We systematically searched MEDLINE, PubMed, CINAHL, EMBASE, and reference lists of included articles, in June 2016. We included all types of peer-reviewed designs on the five topics. Couples of two independent reviewers performed the selection process, the quality assessment, and data extraction.

Results: We included 67 studies with low to moderate quality. Non-conveyance rates for general patient populations ranged from 3.7\%-93.7\%. Non-conveyed patients have a variety of initial complaints, common initial complaints are related to trauma and neurology. Furthermore, vulnerable patients groups as children and elderly are more represented in the non-conveyance population. Within $24 \mathrm{~h}-48 \mathrm{~h}$ after non-conveyance, $2.5 \%-6.1 \%$ of the patients have EMS representations, and $4.6-19.0 \%$ present themselves at the ED. Mortality rates vary from 0.2\%-3. $5 \%$ after $24 \mathrm{~h}$, up to $0.3 \%-6.1 \%$ after $72 \mathrm{~h}$. Criteria to guide non-conveyance decisions are vital signs, ingestion of drugs/alcohol, and level of consciousness. A limited amount of non-conveyance guidelines or protocols is available for general and specific patient populations. Factors influencing the non-conveyance decision are related to the professional (competencies, experience, intuition), the patient (health status, refusal, wishes and best interest), the healthcare system (access to general practitioner/other healthcare facilities/patient information), and supportive tools (online medical control, high risk card).

Conclusions: Non-conveyance rates for general and specific patient populations vary. Patients in the nonconveyance population present themselves with a variety of initial complaints and conditions, common initial complaints or conditions are related to trauma and neurology. After non-conveyance, a proportion of patients reenters the emergency healthcare system within 2 days. For ambulance professionals the non-conveyance decisionmaking process is complex and multifactorial. Competencies needed to perform non-conveyance are marginally described, and there is a limited amount of supportive tools is available for general and specific non-conveyance populations. This may compromise patient-safety.
\end{abstract}

Keywords: Emergency medical services [MeSH], Patient safety [MeSH], Clinical competence [MeSH], Nonconveyance

\footnotetext{
* Correspondence: Remco.Ebben@han.nl

${ }^{1}$ Research Department of Emergency and Critical Care, HAN University of Applied Sciences, Faculty of Health and Social Studies, PO Box 6960, 6503, GL, Nijmegen, The Netherlands

Full list of author information is available at the end of the article
} 


\section{Background}

The past decades, ambulance care has evolved from a health care facility that conveys patients to the hospital, into emergency medical services (EMS) that provide advanced out-of-hospital care for (non-) life-threatening conditions [1, 2]. At the same time, the utilization of ambulance care has increased throughout the developed world, with various underlying reasons such as ageing of the population, changes in social support, accessibility and costs [3]. Together, these developments put a growing demand on ambulance systems and ambulance capacity, the emergency departments (ED) and the wider healthcare system, and this may compromise patient safety, healthcare quality, and access [3]. In addition to this growing demand, frequent overcrowding of the ED occurs $[4,5]$.

The ambulance process is situated within this context. This process often results in patient conveyance to an ED or other healthcare facility, but ambulance care can also result in patients not being conveyed. The NHS Litigation Authority (2012) defines conveyance as "the transfer of patients, medical and clinical personnel, equipment and associated records, as appropriate including from one healthcare facility to another as well as the initial journey from the scene." [6]. Non-conveyance is defined as "an ambulance deployment as appropriate, where the patient after examination and/or treatment on-scene does not require conveyance with medical personnel and equipment to the healthcare facility" [7]. Non-conveyed patients can be treated and 'discharged' on-scene, or may be referred to other (primary) healthcare facilities such as the general practitioner. According to the literature, non-conveyance can be divided in two categories: the patient-initiated refusal and the ambulance professional decision [8]. Often, non-conveyance is a combination of these two categories.

Non-conveyance rates of patients who received onscene emergency care from an ambulance emergency crew, have been reported up to $30 \%[9,10]$. On the other hand, it has been estimated that $11 \%-61 \%$ of the conveyances is medically not necessary [11]. Factors influencing these non-conveyance rates are patients with low-acuity problems or primary care problems who call an ambulance [12, 13], accuracy of triage systems at the EMS dispatch centre [14], and professional competencies [15].

The priority to conduct research on non-conveyance is reflected on the Dutch National Pre-hospital Research Agenda for EMS 2014-2018 [16]. From patient-safety and professional perspective, little is known about nonconveyance. Insight into characteristics and outcomes of the non-conveyance patients is lacking. Furthermore, it is unknown how often non-conveyance exactly occurs, which complaints non-conveyed patients have, what care is provided after non-conveyance, and how often these patients have adverse events. Conversely from the professional perspective, little is known about the on-scene nonconveyance decision-making process. As ambulance care has become a more complex environment, ambulance professionals are faced with decision-making over multiple care options as conveyance to an emergency department, or another non-emergency service, treat-and-release or referral to another healthcare professional [17]. Literature described that this decision-making process requires adequate competencies, skills and clinical reasoning of ambulance professionals [18], although ambulance professionals curricula include a little on conveyance decision making [19]. Also, few ambulance services developed non-conveyance protocols and policies [20]. However, the question is whether the literature describes guidelines, protocols or triage criteria to support the ambulance professionals in the decision making process for non-conveyance, how competent are they to decide and apply for non-conveyance, and how are they influenced during the decision making process for nonconveyance? These aspects of patient safety and ambulance professional perspectives related to non-conveyance in ambulance EMS have not yet been synthesized in an overview.

\section{Aim}

The aim of this systematic review is twofold. The first aim is safety orientated, as we want to describe nonconveyance rates, characteristics of patients, and followup care after non-conveyance. The second aim is formulated from the perspective of the ambulance professional, as we want to describe available guidelines or protocols and triage criteria, competencies needed by ambulance professionals to make appropriate (non-) conveyance decisions, and also to describe which factors influence ambulance professionals during the decisionmaking process.

\section{Methods \\ Design}

A systematic review of the literature was performed according to the steps of the Cochrane Handbook for Systematic Reviews of Interventions [21]. This review is reported in concordance with the Preferred Reporting Items for Systematic reviews and Meta-Analyses (Additional file 1: PRISMA) statement [22].

\section{Search strategy}

Firstly, the Cochrane database for systematic reviews and the DARE database were checked for a similar review (protocol). No review was identified, therefore systematic searches were performed in MEDLINE (EBSCO), PubMed, CINAHL (EBSCO), and EMBASE (OVID) in June 2016. Search strategies were developed to represent 'terms for non-conveyance' AND 'terms for pre-hospital ambulance care'. Full search strategies per database are given in Additional file 2: Appendix 1 . 
Searches were not restricted by year of publication. In addition to the electronic searches, after full-text inclusion we hand-searched reference lists to identify relevant studies.

\section{Study selection procedure}

We included all types of peer-reviewed systematic reviews, and quantitative or qualitative designs in real clinical practice or simulation situations, on nonconveyance. We defined non-conveyance as 'the situation where an ambulance was dispatched and where the patient received on-scene diagnostics and/or treatment, followed by professional and/or patient initiated non-conveyance to the ED or another emergency care facility'. Studies were included when reporting on one or more of the following criteria:

- Non-conveyance rates;

- Characteristics of non-conveyed patients;

- Follow-up care after non-conveyance;

- Non-conveyance guidelines, protocols, or on-scene triage criteria;

- Professional competencies needed to initiate nonconveyance;

- Factors influencing the non-conveyance decisionmaking process.

Conference abstracts, narrative reviews, editorials, personal communications, or unpublished studies were excluded. All articles were screened on title and abstract by two independent reviewers (RE, SB, RS, NT, LV). In case of doubt, a third reviewer (SB, LV) was asked to make a final decision. The remaining articles were screened full text by two independent reviewers (RE, SB, RS, NT, JL, LV). In addition, reference lists of included articles were screened (RE, JL) and potentially relevant publications were screened in a similar way (RE, RS, NT, JL).

\section{Quality assessment}

To assess the risk of bias of (pre-, or quasi-) experimental studies we used the 'risk of bias assessment tool' [21]. This tool is a domain-based evaluation to assess selection bias, performance bias, attrition bias, detection bias and reporting bias. For non-randomized studies, the Cochrane collaboration recommends to add additional domains. Therefore, we added two domains to the tool: (1) randomization (yes/no), and (2) control group (yes/ no). To assess the quality of systematic reviews we used AMSTAR, as recommended by Cochrane [23]. To assess the quality of observational studies (retrospective, crosssectional, prospective) and qualitative studies we used tools developed for evaluating primary research papers in a variety of fields [24]. From the 14-criteria quantitative tool, we deleted three criteria (criteria five, six, and seven) on experimental research as we assessed quality of experimental studies with the tool described above. For qualitative studies we used the 10-criteria tool. The quality assessment was performed by couples of two independent researchers (RE, RS, NT, JL). In case of doubt, a third reviewer from these four researchers was asked to make a final decision.

\section{Data extraction}

Data were extracted by two independent researchers (RE, RS, NT, JL). Outcomes extracted were non-conveyance rates, characteristics of non-conveyed patients, existing guidelines, protocols or triage criteria for non-conveyance, follow-up care by patients after non-conveyance, ambulance professionals competencies needed to perform nonconveyance, and factors influencing ambulance professionals during the non-conveyance decision-making process.

\section{Data synthesis and presentation}

Due to heterogeneity of the studies with regard to patient populations, interventions and outcomes, a metaanalysis was not possible. Instead, we extensively analysed and synthesized the studies, by scrutinizing and categorizing data and formulating (sub)themes. To report non-conveyance rates, percentages were extracted or calculated. When patients died or left the scene before ambulance arrival, these were not taken into account for non-conveyance rates. To compare patients' initial complaints or conditions across studies, we classified these according to the ICD-10 classification [25]. The ICD-10 classification is an international standard to classify diseases or other health problems, and is widely accepted and used. For each ICD-10 category we described the proportions of the patients who had a certain classification.

\section{Results}

\section{Review statistics}

The initial search identified 2989 unique records, after the selection procedure 67 studies were included (see Fig. 1). A list of excluded articles $(n=67)$ is provided in Additional file 3: Appendix 2.

\section{Study Characteristics}

The designs of the included studies concerned two systematic reviews $[10,26]$, four experimental designs: one cluster-randomized controlled trial [27], one quasiexperimental [28], and two pre-test post-test [29, 30], 52 observational designs: 27 retrospective [8, 9, 31-55], 23 prospective [56-78], and two cross-sectional [79, 80], one mixed method design [81], and eight qualitative designs [82-89] (Table 1 and Table 2). 


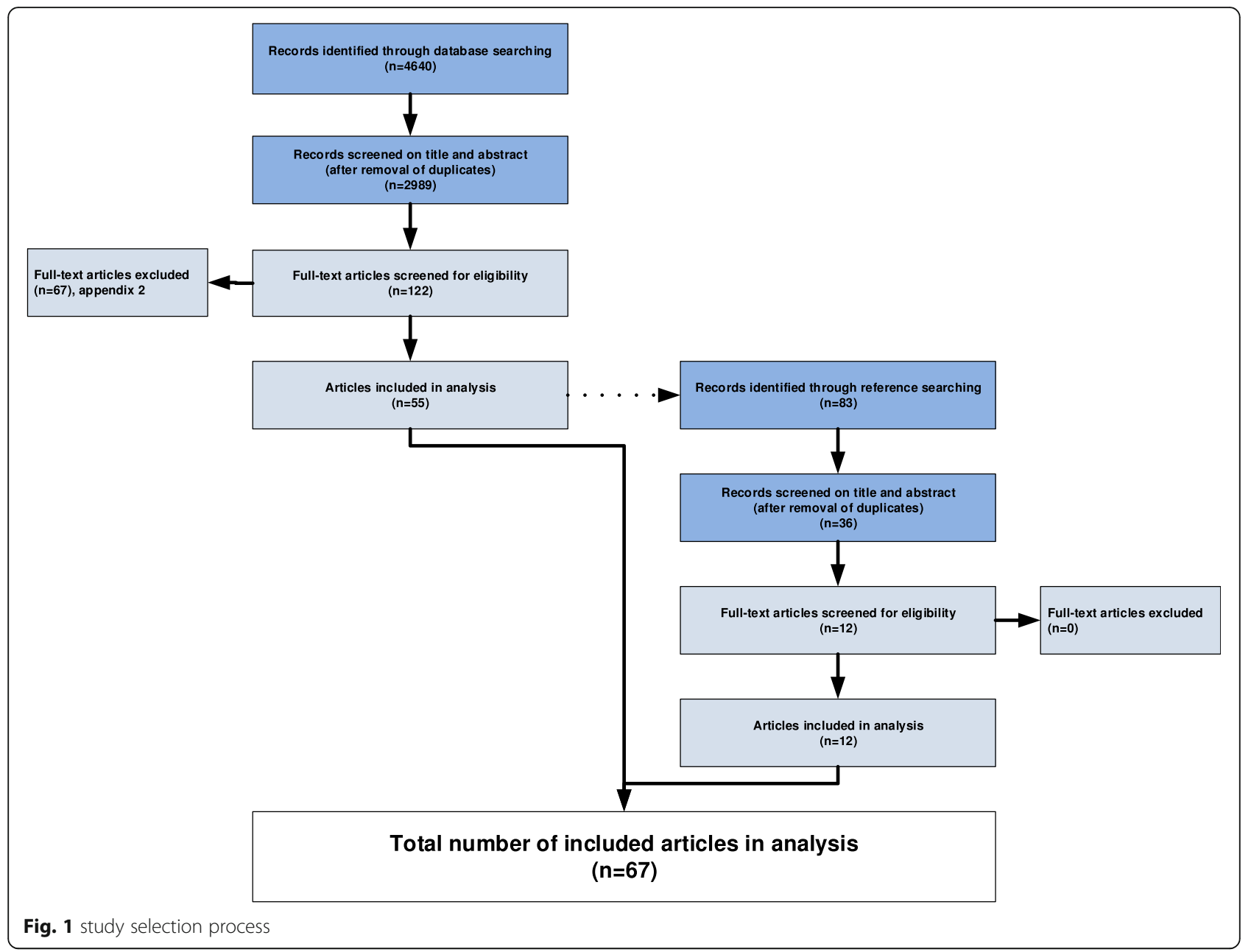

The two systematic review were performed in Australia and the UK. The empiric studies were conducted in North America $(n=36)$, Europe $(n=17)$, Australia $(n=6)$, Asia $(n=5)$, and Africa $(n=1)$, and concerned general patient populations or specific patient populations, including patients with hypoglycaemia, patients who refused conveyance, paediatric and/or older patients, patients with supraventricular tachycardia, patient with acute opioid overdose, post-ictal patients, and patients who had fallen. The ambulance professionals in these studies were ambulance nurses, basic and advanced life support paramedics, emergency medical technicians, (specialized) physicians, general practitioners, and first responder fire fighters. For this review we will use the term 'ambulance professional' to cover all these types of professionals.

\section{Quality assessment (Additional file 4: Appendix 3,} Additional file-5: Appendix 4, Additional file 6: Appendix 5, Additional file 7: Appendix 6)

The two included systematic reviews had moderate [26] and low quality [10] (Additional file 4: Appendix 3). The four experimental designs included one CRCT of moderate quality [27], one quasi-experimental study [28] and two pre-test post-test $[29,30]$ of poor quality (Additional file 5: Appendix 4). The quality of the quantitative studies $(n=53)$ varied from good [76] to poor [42] (Additional file 6: Appendix 5), and the quality of the qualitative studies $(n=8)$ varied from good [83] to poor [88] (Additional file 7: Appendix 6).

\section{Outcomes}

\section{Non-conveyance rates (Additional file 8: Appendix 7)}

Non-conveyance was initiated by the ambulance professional, the patient and/or his relatives, or a joint decision. Non-conveyance rates for general patient populations ranged from $3.7 \%$ up to $93.7 \%$ [28, 30, 31, 33-35, 37, 38, 40-43, 45, 46, 49, 51, 52, 57, 60, 61, 64, $68,77,81]$. Seventeen studies reported non-conveyance rates for specific patient populations. For patients with hypoglycaemia non-conveyance rates ranged from $12.2 \%$ up to $84.3 \%$ [32, 48, 53, 58, 59, 75]. Non-conveyance rates for people who had fallen ranged from $11 \%-56 \%$ $[26,27,73,74]$. For paediatric patients non-conveyance 


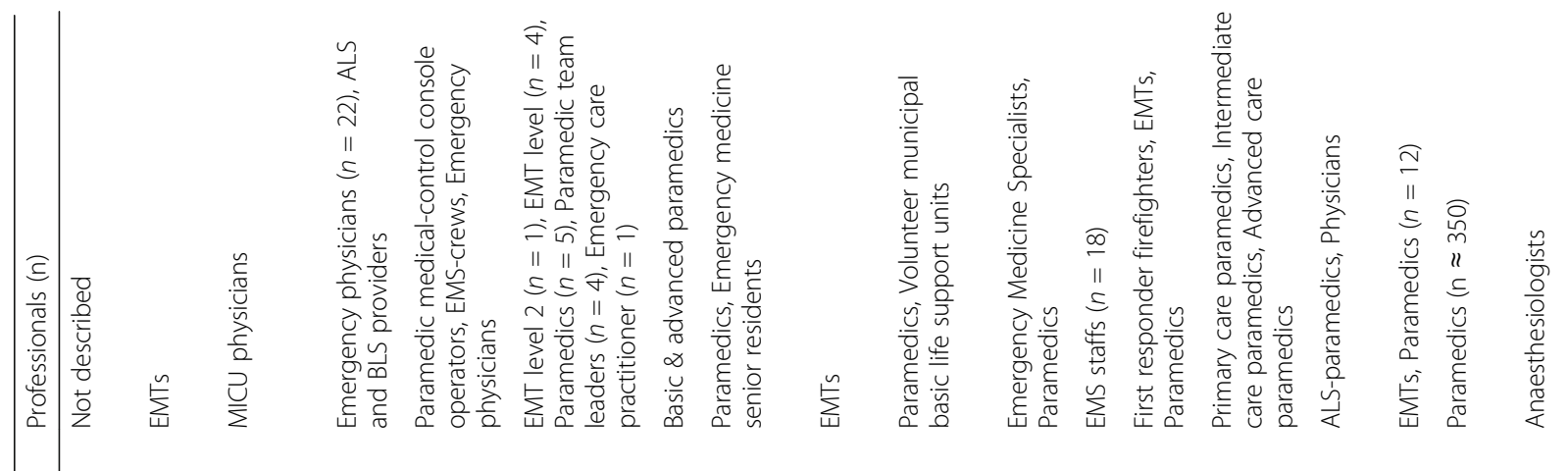



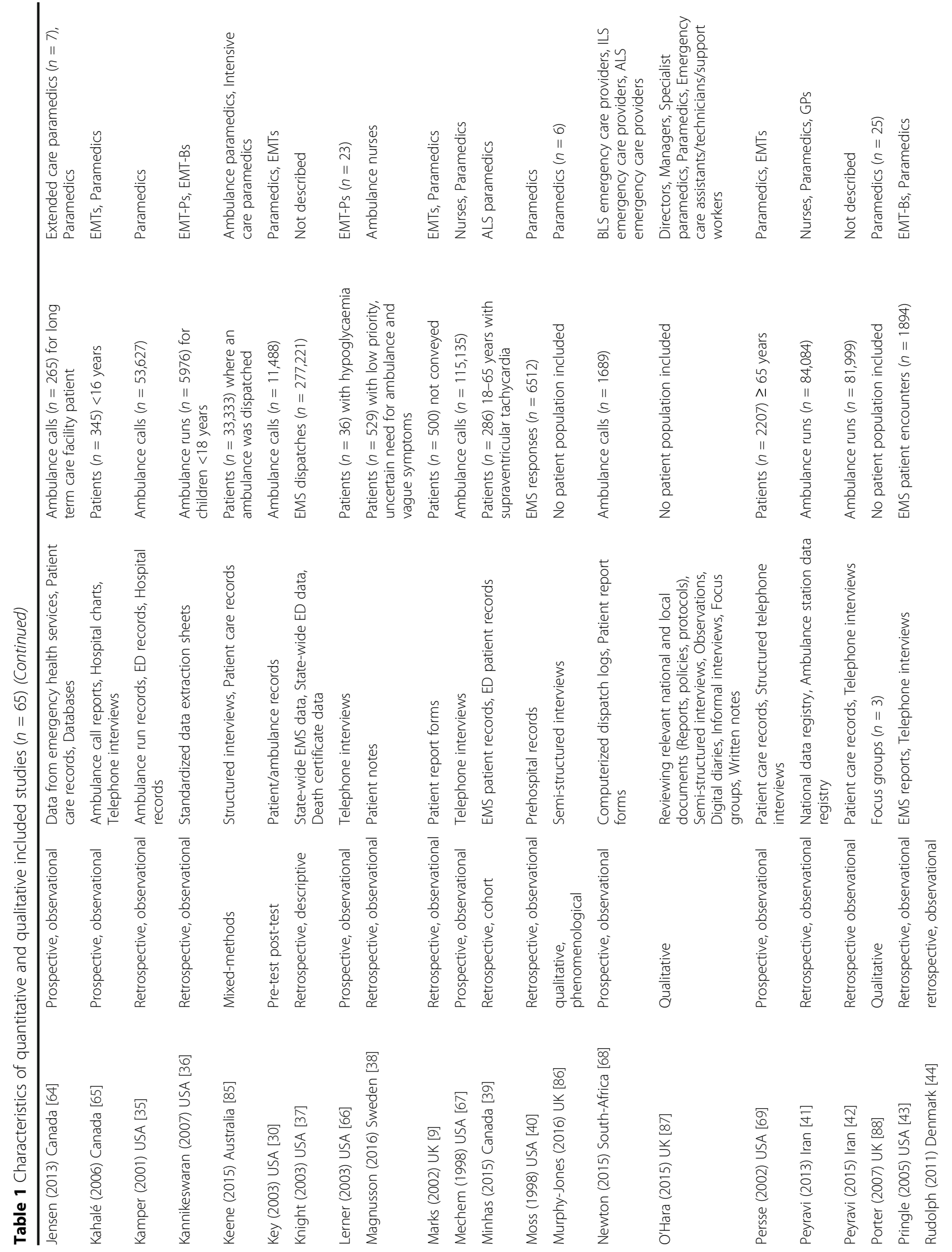


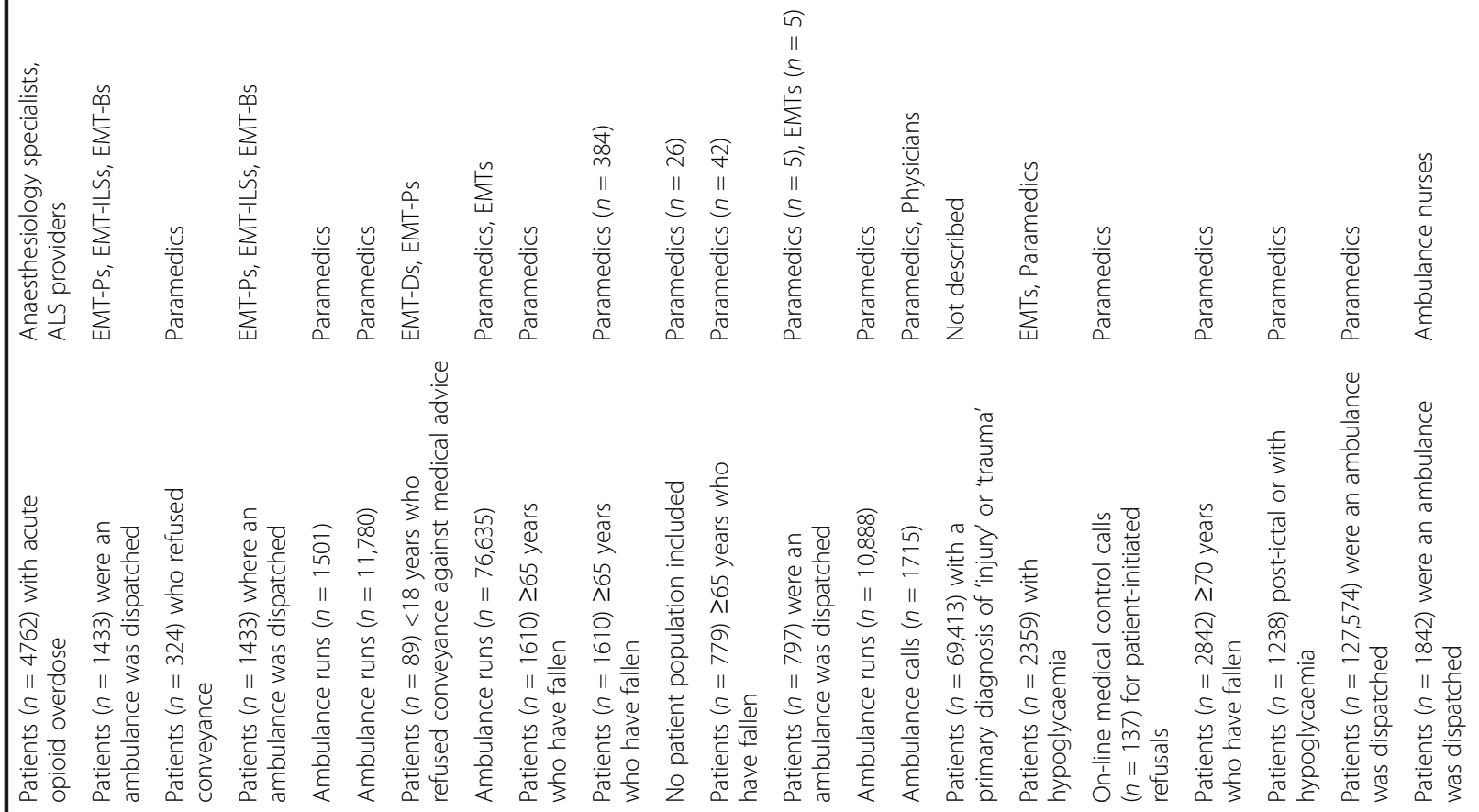
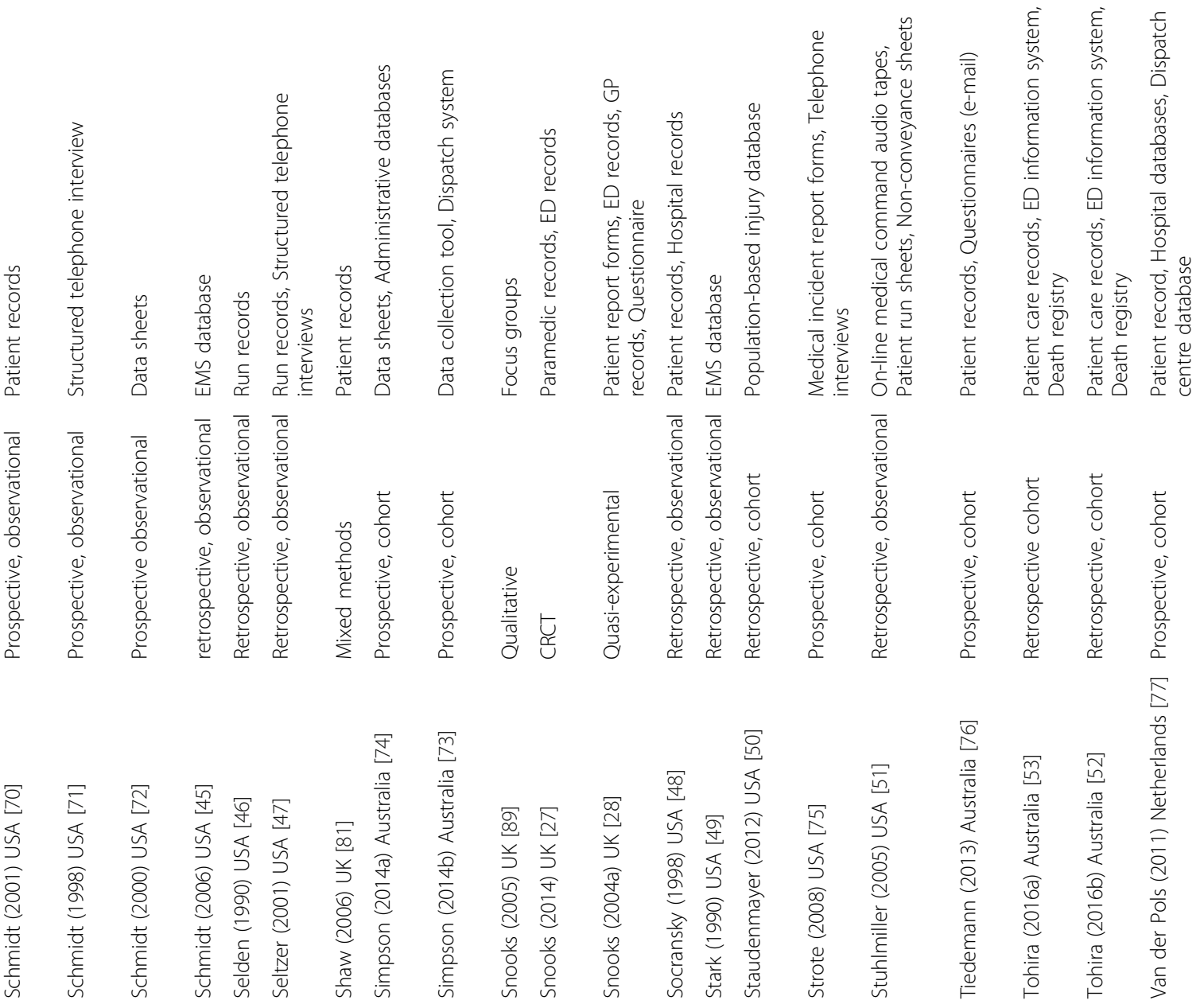

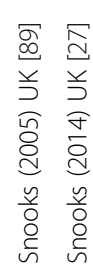
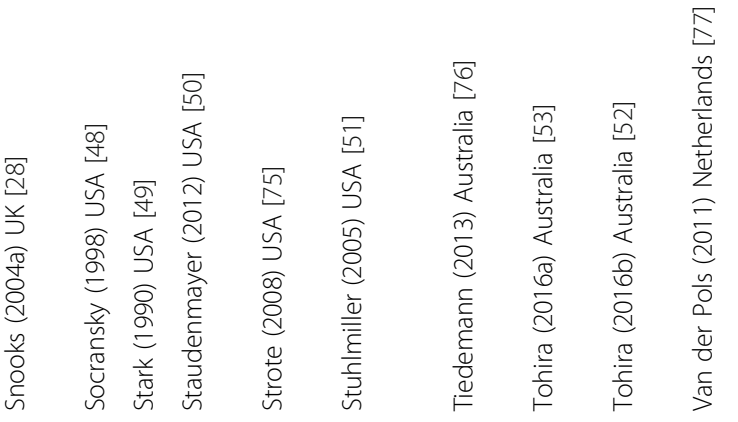


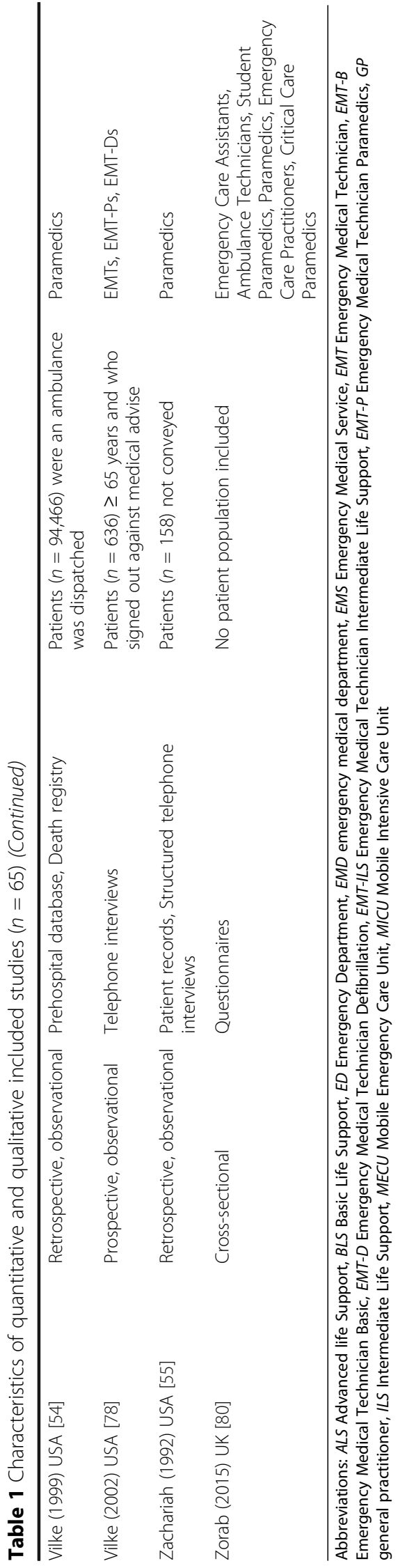


Table 2 Characteristics systematic reviews $(n=2)$

\begin{tabular}{|c|c|c|c|c|}
\hline $\begin{array}{l}\text { 1st author (year) } \\
\text { country }\end{array}$ & Aim & Databases & Selection criteria & Included articles \\
\hline $\begin{array}{l}\text { Mikolaizak (2013) } \\
\text { Australia [26] }\end{array}$ & $\begin{array}{l}\text { To summarize the evidence in } \\
\text { relation to (1) non-conveyance } \\
\text { rates, (2) outcomes following } \\
\text { non-conveyance, and ( } 3 \text { ) outcomes } \\
\text { from alternative care pathways } \\
\text { for non-conveyed older people } \\
\text { who have fallen }\end{array}$ & $\begin{array}{l}\text { 1. Medline } \\
\text { 2. Embase } \\
\text { 3. CINAHL } \\
\text { 4.PsycINFO } \\
\text { 5.Cochrane Library } \\
\text { 6. Web of Science }\end{array}$ & $\begin{array}{l}\text { 1. Peer-reviewed articles } \\
\text { 2. Original data relating to } \\
\text { non-transport rates for older } \\
\text { people who have fallen } \\
\text { 3. Outcomes on falls or outcomes } \\
\text { for alternate care pathways for } \\
\text { non-transported people who } \\
\text { have fallen }\end{array}$ & $\begin{array}{l}12 \text { articles: } 2 \text { randomized } \\
\text { controlled trials, } 5 \text { prospective } \\
\text { cohort studies, } 4 \text { retrospective } \\
\text { cohort studies and } 1 \text { historical } \\
\text { cohort trial. }\end{array}$ \\
\hline $\begin{array}{l}\text { Snooks (2004b) } \\
\text { UK [10] }\end{array}$ & $\begin{array}{l}\text { 1. To describe outcomes of } \\
\text { non-conveyed patients } \\
\text { 2. To describe triage ability of crews } \\
\text { 3. To assess effectiveness and safety of } \\
\text { protocols that allow crews to convey } \\
\text { patients to alternative receiving units } \\
\text { or to self-care }\end{array}$ & $\begin{array}{l}\text { 1. Medline } \\
\text { 2. BIDS } \\
\text { 3. Healthplan } \\
\text { 4. Helmis }\end{array}$ & $\begin{array}{l}\text { Articles on paramedics trained } \\
\text { with extra skills to perform tasks } \\
\text { beyond their baseline competencies }\end{array}$ & $\begin{array}{l}31 \text { articles: } 13 \text { retrospective } \\
\text { observational studies, } 8 \\
\text { prospective observational } \\
\text { studies, } 6 \text { cross-sectional studies, } \\
3 \text { case studies and } 1 \text { quasi- } \\
\text { experimental study }\end{array}$ \\
\hline
\end{tabular}

rates ranged from $13.2 \%-27.7 \%[36,62,79]$. Two studies reported non-conveyance rates for patients with an opioid overdose, ranging from $6.0 \%-77.0 \%[44,54]$. Nonconveyance rates for other specific patient groups were 14.0\% for post-ictal patients [53], 33.2\% for patients with supraventricular tachycardia [39], 10.7\%-11.5\% for elder people [69], and 8.6\% for patients with injuries [50].

\section{Characteristics of non-conveyed patients (Additional file 8 Appendix 7)}

The demographic characteristics were age, gender, ethnicity, and geographic area. For general patient populations, the age ranges from 14 up to 90 years $[9,29,31$, $33,38-40,45,48,50,52,54,56,62,63,65-67,73,74$, $76,78,79,85]$. Twenty studies reported on patient gender: in ten studies the gender is predominantly male, in the other studies the population is predominantly female $[9,33,38-40,45,48,50,52,54,62,63,65-67,73,74$, $76,79,85]$. Three studies described the geographic location of non-conveyed patients [33, 65, 74]. Two of these show that most non-conveyed people stay in a metropolitan/urban area. The third study showed that $58.6 \%$ of the patient are in their residence. Two studies described the patient's ethnicity $[45,79]$, with one study reporting $90.6 \%$ of the non-conveyed patient as white, the other study reported $48.3 \%$ of the patient as AfricanAmerican.

The clinical characteristics of the patient were initial complaints and conditions, vital signs, and patient history. A variety of initial complaints and conditions was described $[9,29,34,38,40,45,52,56,57,61-63,65,74$, $77-79,85]$. Most often, we found initial complaints and conditions classified as VI-diseases of the nervous system $(n=16)$ or category XX - External causes of morbidity and mortality $(n=16)$. For category $V I$ the proportion of patients with these complaints and conditions ranged from $1.0 \%-29.0 \%[9,29,34,38,40,45,52$, $56,57,61,63,65,77-79,85]$, for category $X X$ the proportion ranged from $11.0 \%-68.5 \%[9,29,38,40,45$, $52,56,57,61-63,65,77-79,85]$.

Three studies described the vital signs of nonconveyed patients $[50,52,63]$. One study on a general population reported that $14.9 \%$ of the non-conveyed patients had abnormal vital signs (blood pressure, $\mathrm{O}_{2}$-saturation, Glasgow Coma Scale, and body temperature) [52]. A second study in a non-conveyed general patient population reported that $70.0 \%$ had a blood pressure within normal limits, $72.2 \%$ had a heart rate within normal limits, and $63.2 \%$ had a respiratory rate within normal limits [63]. The last study on vital signs with injured people not conveyed reported a mean systolic blood pressure of $134.7 \mathrm{mmHg}( \pm 21.1)$, a mean pulse rate of $91.8( \pm 15.9)$, and a mean Glasgow Coma Scale of 15.0 $( \pm 0.3)[50]$.

Five studies described the patient's history by describing the medical history and/or current medication use $[48,63,73,74,76]$. Two studies [63, 76] described the medical history, for general patient populations 68.7\% had no medical history [63], for people aged $\geq 70$ years who had fallen $43.8 \%$ had urinary incontinence and $39.0 \%$ had a central nervous system disorder.

\section{Follow-up of patients after non-conveyance (Table 3)}

Follow-up was reported as (a) repeated access to healthcare and (b) patient outcomes. Sixteen studies combined these outcome categories, the other studies used outcomes within one category $[8,26,28,32,37-40,43-45$, $48,50,52,55-59,62,64-67,69,75-78,90]$. Repeated access to healthcare was specified as repeated access to (1) emergency department (2) EMS-system (call or ambulance run), (3) the general practitioner, and (4) walkin clinic. For all outcomes, a variety of follow-up periods was used. In every study that reported on repeated access to healthcare a proportion of patients re-entered the (emergency) healthcare system. 
Table 3 Follow-up care after non-conveyance

\begin{tabular}{|c|c|c|}
\hline 1st author (year) Country [ref] & Follow-up outcomes & Results \\
\hline Anderson (2002) Denmark [32] & $\begin{array}{l}\text { - Patient outcome - hospitalization } \\
\text { - Patient outcome - recurrent symptoms }\end{array}$ & $\begin{array}{l}\text { - } 76 / 968(7.9 \%) \text { patients have secondary blood glucose } \\
\text { regulatory problems }<72 \mathrm{~h} \\
\circ \quad 46 / 76(60.5 \%) \text { have a recurrent hypoglycaemia, 33/46 } \\
(71.7 \%) \text { of these cases occur }<24-72 \mathrm{~h} \\
\text {. } 49 / 968(5.1 \%) \text { are hospitalized }<72 \mathrm{~h} \\
0 \quad 21 / 49(42.9 \%) \text { have a recurrent hypoglycaemia of which } \\
12 / 21(57.1 \%) \text { are hospitalized }<24-72 \mathrm{~h}\end{array}$ \\
\hline Burstein (1996) USA [56] & $\begin{array}{l}\text { - Repeat access general healthcare - GP } \\
\text { - Repeat access emergency healthcare - } \\
\text { EMS (call or EMS run) } \\
\text { - } \\
\text { Repeat access emergency healthcare - ED }\end{array}$ & $\begin{array}{l}\text { - 199/321 (62.0\%) patients who had follow-up. } \\
\text { 95/199 (47.7\%) patients sought additional medical care < } \\
1 \text { week. } \\
\text { - } 51 / 95 \text { (53.7\%) went to the ED: } 7 \text { through EMS, } 41 \text { referred } \\
\text { themselves to the ED and } 3 \text { were referred by their physician. } \\
\text { - } 44 / 95 \text { (46.3\%) were seen by their physician. }\end{array}$ \\
\hline Burstein (1998) USA [57] & 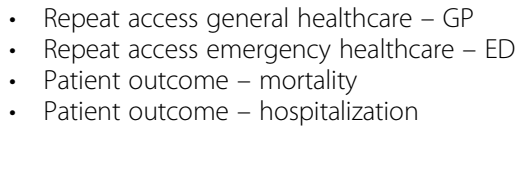 & 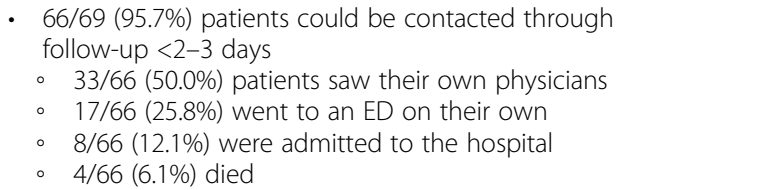 \\
\hline
\end{tabular}

Cain (2003 USA [58] (call or EMS run)
Carter (2002) Canada [59]

Cone (1995) USA [8]

Haines (2006) USA [62]

Højfeld (2014) Denmark [34]

Jensen (2013) Canada [64]

Kahalé (2006) Canada [65]
- Repeat access emergency healthcare - EMS
- Repeat access general healthcare - GP

- Repeat access emergency healthcare - ED

- Patient outcome - hospitalization
- Patient outcome - recurrent symptoms

- Repeat access general healthcare - GP

- Patient outcome - hospitalization
- Repeat access emergency healthcare - ED

40/145 (27.6\%) patients had signs and symptoms compatible with low blood sugar occurring $<10$ months after initial event and requiring a repeat EMS call:

- 2/24 (8.3\%) patients $>65$ years

- 38/121 (31.4\%) patients < 65 years

$3 / 145$ (2.1\%) patients had signs and symptoms compatible with low blood sugar occurring $<48 \mathrm{~h}$ after initial event and requiring a repeat EMS call:

- $0 / 24(0.0 \%)$ patients $>65$ years

- $3 / 121$ (2.5\%) patients <65 years

- No significant differences in repeat $(p=.43)$ any time during the ten-month study period, recurrences $(p=.33)<48$ $\mathrm{h}$ and interval for repeat episodes $(p=.60)$ between conveyed and non-conveyed patient calls.

Repeated access to healthcare $<21$ days:

- $6 / 41$ (14.6\%) patients for all complaints

- $2 / 41$ (4.9\%) patients for the same complaint

54/81 (67\%) had follow-up:

- 37/54 (68.5\%) sought no medical care

- 10/54 (18.5\%) were evaluated in the ED: 3 were

discharged, 7 were admitted: 3 were admitted to monitored beds and 4 were admitted to unmonitored beds

- $7 / 54$ (13.0\%) saw their own physician $<48 \mathrm{~h}$ after refusal

527/704 (74.8\%) completed phone follow-up:

- 13/527 (2.5\%) non-transport group hospitalized

- 279/527 (52.9\%) patients had follow-up-care

$<72 \mathrm{~h}$ (median $2.5 \mathrm{~h}$, inter-quartile range 1.5-13 h)

- 203/279 (72.6\%) patients had follow-up-care <12 h

- 148/279 (65.9\%) patients came to ED

- 95/279 (34.1\%) patients came via primary care physician

- 19/279 (6.8\%) patients were evaluated by a medical provider more than once in $72 \mathrm{~h}$

$113 / 1609(7.0 \%)$ patients had renewed treatment in hospital or $\mathrm{ED}<24 \mathrm{~h}$

- 58/113 (51.3\%) had to be admitted

- $51 / 113(45.1 \%)$ visited the ED

- $4 / 113(3.5 \%)$ died

- Patient outcome - mortality

- Patient outcome - hospitalization

- Repeat access emergency healthcare - EMS (call or EMS run)

- Repeat access general healthcare - GP

- Repeat access general healthcare - walk-in clinic

- Repeat access emergency healthcare - ED
6/238 (2.5\%) patients who received extended paramedic care but who were not transported subsequently triggered a EMS call $<48 \mathrm{~h}$

51/345 (14.8\%) non-transported children were seen at the ED $<48 \mathrm{~h}$

Telephone follow-up with patients $(n=106)$ about additional care $<48 \mathrm{~h}$ : 
Table 3 Follow-up care after non-conveyance (Continued)

- 51/106 (48.1\%) patients did not seek medical follow-up

- $28 / 106$ (26.4\%) patients went to the ED

- 22/106 (20.8\%) patients visited the family physician/ paediatrician office

- $4 / 106$ (3.8\%) patients visited a walk-in clinic

- $1 / 106(0.9 \%)$ patients went to a hospital/outpatient clinic

Knight (2003) USA [37]

Lerner (2003) USA [66]

Magnusson (2016) Sweden [38]

Mechem (1998) USA [67]

Minhas (2015) Canada [39]

Moss (1998) USA [40]

Persse (2002) USA [69]
- Repeat access emergency healthcare - ED

- Repeat access emergency healthcare - EMS (call or EMS run)

- Patient outcome - mortality

- Patient outcome - hospitalization
3454/26574 (13.0\%) follow-up was obtained <1 week:

- $174 / 3454(5.0 \%)$ patients were admitted to the hospital

- $25 / 3454(0.7 \%)$ patients died

- 465/3454 (13.5\%) patient had an EMS dispatch

- < 3 years: $8 / 465(1.7 \%)$

- 3-12 years: $14 / 465(3.0 \%)$

- 13-17 years: $24 / 465(5.2 \%)$

- 18-64 years: 301/465 (64.7\%)

- $\geq 65$ years: $118 / 465$ (25.4\%)

- $2790 / 3454(80.1 \%)$ of the patients had an ED visit

- < 3 years: $133 / 3454(3.9 \%)$

- 3-12 years: $175 / 3454(5.1 \%)$

- 13-17 years: $223 / 3454(6.5 \%)$

- 18-64 years: 2041/3454 (59.1\%)

- $\quad \geq 65$ years: $218 / 3454$ (6.3\%)

- $174 / 3454(5.0 \%)$ of the patients were admitted

- < 3 years: $12 / 174(6.9 \%)$

- 3-12 years: $13 / 174(7.5 \%)$

- 13-17 years: $7 / 174(4.0 \%)$

- 18-64 years: 97/174 (55.7\%)

- $\geq 65$ years: $45 / 174$ (25.9\%)

20/36 (55.6\%) sought further medical assistance <48 h:

- Repeat access general healthcare - GP

- Repeat access emergency healthcare - ED

- $11 / 20(55.0 \%)$ called their personal physician

- $8 / 20(40.0 \%)$ visited their personal physician

- $1 / 20$ (5.0\%) went to the ED

38/200 (19.0\%) patients visited the ED $<72 \mathrm{~h}$ :

- Repeat access general healthcare - GP

- Repeat access emergency healthcare - ED

- Patient outcome - hospitalization

24/38 (63.2\%) self to ED

- 12/24 (50.0\%) admitted

- $14 / 38(36.8 \%)$ referred by GP

- 8/14 (57.1\%) admitted

- Repeat access general healthcare - GP

94/103 (91.3\%) patients had no recurrence of symptoms

- Repeat access emergency healthcare - ED in $<72 \mathrm{~h}:$

- Repeat access emergency healthcare - EMS - $7 / 94(7.4 \%)$ contacted private physician (call or EMS run)

- Patient outcome - hospitalization 9/103 (8.7\%) recontacted the EMS $<72 \mathrm{~h}$ :

- $5 / 9(55.6 \%)$ transported and released from ED

- 3/9 (33.3\%) transported and admitted

- $1 / 9(11.1 \%)$ refused transport

Mikolaizak (2013) Australia [26] • Repeat access general healthcare - GP

Follow-up periods varied from 1 to 12 months. Outcomes:

- Repeat access general healthcare - walk-in clinic

12\%-49\% readmission in ambulance or other health service facility, non-transported patients have significantly higher risk of death compared to age matched peers

- Repeat access emergency healthcare - ED

- Repeat access emergency healthcare - EMS (call or EMS run)

- Patient outcome - mortality

- Patient outcome - hospitalization

- Repeat access emergency healthcare - EMS (call or EMS run)

$1 / 76(1.3 \%)$ of the patients treated and released had 14 representations $<72 \mathrm{~h}$

- Repeat access emergency healthcare - ED

- Repeat access emergency healthcare - EMS (call or EMS run)

- Patient outcome - mortality

- Patient outcome - hospitalization

431/443 (97.3\%) patients a follow-up was obtained:

- 10/431 (2.3\%) called EMS again <48 h

- $4 / 10$ (40.0\%) were admitted to a hospital

- $4 / 10(40.0 \%)$ were discharged from the ED

- $1 / 10(10.0 \%)$ died

- $1 / 10(10.0 \%)$ was transferred to another facility

- Patient outcome - hospitalization
Phase 1: 151/254 (59.5\%) patients were contacted by telephone:

- 56/151 (37.1\%) sought further medical help $<24 \mathrm{~h}$

- 19/151 (12.6\%) were hospitalized Phase 2: 109/198 (55.1\%) patients were contacted by telephone: 
Table 3 Follow-up care after non-conveyance (Continued)

\begin{tabular}{|c|c|c|}
\hline & & $\begin{array}{l}\cdot \text { 37/109 (33.9\%) sought further medical help <24 h } \\
\text { - } 7 / 109 \text { (6.4\%) were hospitalized }\end{array}$ \\
\hline Pringle (2005) USA [43] & $\begin{array}{l}\text { - Patient outcome - mortality } \\
\text { - Patient outcome - hospitalization }\end{array}$ & $\begin{array}{l}\text { 310/906 (34.2\%) follow-up was obtained (1 week): } \\
\text { • } 172 / 310(55.5 \%) \text { patients sought medical care: } \\
\text { o } 106 / 172(61.6 \%) \text { medical care was changed } \\
\text {. } 25 / 310(8.1 \%) \text { were admitted to a hospital } \\
\text { - } 1 / 310(0.3 \%) \text { patients died }\end{array}$ \\
\hline Rudolph (2011) Denmark [44] & - Patient outcome - mortality & 18/2241 (0.8\%) patients released on scene died $<48 \mathrm{~h}$ \\
\hline Schmidt (2006) USA [45] & - Patient outcome - mortality & 2/128 (1.6\%) patients not-transported died $<30$ days \\
\hline Snooks (2004a) UK [28] & - Patient outcome - hospitalization & $\begin{array}{l}\text { Intervention group: } 5 / 93 \text { (5.4\%) patients were admitted to a } \\
\text { hospital <14 days } \\
\text { Control group: } 12 / 195 \text { (6.2\%) patients were admitted to a } \\
\text { hospital }<14 \text { days }\end{array}$ \\
\hline Socransky (1998) USA [48] & $\begin{array}{l}\text { - Repeat access emergency healthcare - ED } \\
\text { - Patient outcome - hospitalization } \\
\text { - Patient outcome - recurrent symptoms }\end{array}$ & $\begin{array}{l}25 / 412(6.1 \%) \text { of the patients who refused transport had a } \\
\text { relapse < } 48 \text { h: } \\
\text { - } 14 / 25(56.0 \%) \text { refused transport again } \\
\text { - } 6 / 25(24.0 \%) \text { admitted to the ED } \\
\text { - } 5 / 25(20.0 \%) \text { were admitted to a hospital }\end{array}$ \\
\hline Staudenmayer (2011) USA [50] & $\begin{array}{l}\text { - Repeat access emergency healthcare - ED } \\
\text { - Patient outcome - hospitalization } \\
\text { - Patient outcome - mortality }\end{array}$ & $\begin{array}{l}\text { 1715/5865 (29.2\%) follow-up obtained: } \\
\text { • } 1616 / 1715(94.2 \%) \text { patients were seen in the ED and } \\
\text { discharged } \\
\text {. } 92 / 1715(5.4 \%) \text { were admitted to the hospital } \\
\text {. } 7 / 1715(0.4 \%) \text { died }\end{array}$ \\
\hline Strote (2008) USA [75] & $\begin{array}{l}\text { - Repeat access general healthcare - GP } \\
\text { - Repeat access emergency healthcare - ED } \\
\text { - Patient outcome - hospitalization }\end{array}$ & $\begin{array}{l}\text { 203/402 (49.5\%) follow-up obtained: } \\
\text { • } 111 / 203(54.7 \%) \text { patients contacted their primary care } \\
\text { physician <24 h } \\
\text { • } 8 / 203(3.9 \%) \text { patients called the EMS again }<48 \mathrm{~h} \\
\cdot \text { - } 16 / 203(7.9 \%) \text { patients went to the hospital }<48 \mathrm{~h}\end{array}$ \\
\hline Tiedemann (2013) UK [76] & - Patient outcome - recurrent symptoms & $\begin{array}{l}62 / 251 \text { ( } 24.7 \% \text { ) of the non-transported patients required } \geq 1 \\
\text { fall related repeat ambulance attendance }<6 \text { months }\end{array}$ \\
\hline Tohira (2016b) Australia [52] & $\begin{array}{l}\text { - Repeat access emergency healthcare - ED } \\
\text { - Repeat access emergency healthcare - EMS } \\
\text { (call or EMS run) } \\
\text { - Patient outcome - mortality } \\
\text { - Patient outcome - hospitalization }\end{array}$ & 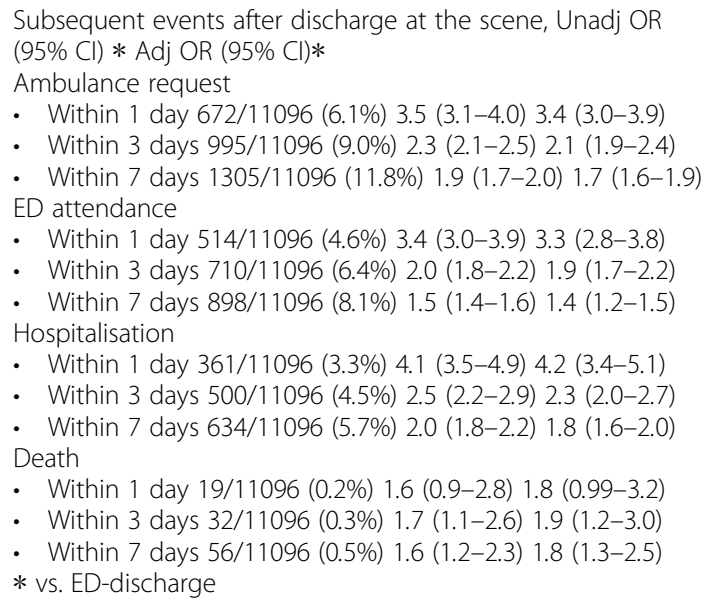 \\
\hline $\begin{array}{l}\text { Van der Pols (2011) The } \\
\text { Netherlands [77] }\end{array}$ & - Repeat access general healthcare - GP & 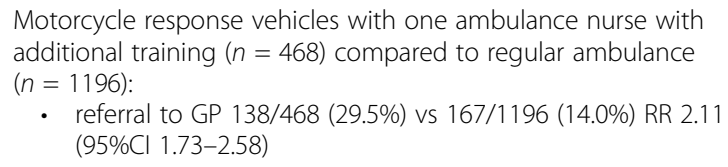 \\
\hline Vilke (2002) USA [78] & $\begin{array}{l}\text { - Repeat access general healthcare - GP } \\
\text { - } \text { Repeat access general healthcare - walk-in } \\
\text { clinic } \\
\text { - Repeat access emergency healthcare - ED } \\
\text { - } \text { Repeat access emergency healthcare - EMS } \\
\text { (call or EMS run) }\end{array}$ & $\begin{array}{cl}\text { 71/121 (58.7\%) follow-up was obtained: } \\
\text {. } 27 / 71 \text { (38.0\%) visited family physician } \\
\text {. } 25 / 71(35.2) \text { visited urgent care facility } \\
\text {. } 9 / 71(12.7 \%) \text { second EMS call and transported to ED } \\
\text {. } 9 / 71(12.7 \%) \text { transport to ED by private vehicle } \\
\text { - } 1 / 71(1.4 \%) \text { second EMS call and treated at scene }\end{array}$ \\
\hline Zachariah (1992) USA [55] & $\begin{array}{l}\text { - Repeat access general healthcare - GP } \\
\text { - Patient outcome - hospitalization }\end{array}$ & $\begin{array}{l}\text { 93/158 (58.9\%) follow-up was obtained: } \\
\text { • } 60 / 93(64.5 \%) \text { sought care from a physician: } \\
\quad 15 / 60(25.0 \%) \text { were admitted to hospital. }\end{array}$ \\
\hline
\end{tabular}


Repeated access to the ED is measured in seventeen studies $[8,26,37,38,40,48,50,52,56,57,62,65-67$, $75,78,90]$. For general patient populations, the followup periods ranged from $<24 \mathrm{~h}$ up to $<7$ days, and repeated access percentages varied from $4.6-7.0 \%(<24 \mathrm{~h})$, $19.0 \%(<48 \mathrm{~h}), \quad 6.4-25.8 \%(72 \mathrm{~h})$ up to $8.1-80.1 \%$ $(<7$ days). For specific patient populations (hypoglycaemia, people who had fallen, people aged $>65$ years, children and people with minor injuries), the follow-up periods ranged from $<48 \mathrm{~h}$ up to $<12$ months, and repeated access percentages varied from 5.0-26.4\% (<48 h), 65.9\% (<72 h), up to $12.0-49.0 \%$ (12 months).

Repeated access to the EMS-system is measured in ten studies [26, 37, 39, 40, 52, 56, 58, 64, 67, 78]. For general patient populations, the follow-up periods ranged from $<24 \mathrm{~h}$ up to $<7$ days, and repeated access percentages varied from $6.1 \%(<24 \mathrm{~h}), 2.3-2.5 \%(<48 \mathrm{~h})$ up to $7.4-$ $13.5 \% \quad(<7$ days). For specific patient populations (hypoglycaemia, people who had fallen, supraventricular tachycardia, and people aged $>65$ years), the follow-up periods ranged from $<48 \mathrm{~h}$ up to $<12$ months, and repeated access percentages varied from $0.0-2.5 \%(<48 \mathrm{~h})$, $1.3-8.7 \%$ (<72 h), 8.3-31.4\% (10 months) up to 12.0 $49.0 \%$ (12 months).

Repeated access to the GP is measured in thirteen studies [8, 26, 38, 55-57, 62, 65-67, 75, 77, 78]. For general patient populations, the follow-up periods ranged from $<48 \mathrm{~h}$ up to $<7$ days, and repeated access percentages varied from $13.0 \%$ ( $<24 \mathrm{~h}$ ), 36.8-50.0\% ( $<72 \mathrm{~h})$ up to $46.2 \%$ ( $<7$ days). For specific patient populations (hypoglycaemia, people who had fallen, children, and people aged $>65$ years), the follow-up periods ranged from $<24 \mathrm{~h}$ up to 12 months, and repeated access percentages varied from $54.7 \%(24 \mathrm{~h}), 7.4-40.0 \%(<48 \mathrm{~h})$, $34.1 \%$ (72 h) up to $12.0-49.0 \%$ (12 months).

Repeated access to walk-in clinic is measured in three studies for specific patient populations (children, people who had fallen, and patients aged $>65$ years) $[26,65,78]$. The follow-up periods used for this outcome ranged from $<48 \mathrm{~h}$ up to 12 months, and repeated access percentages varied from $3.8 \%(<48 \mathrm{~h})$ up to $12.0-49.0 \%$ (12 months).

The patient outcomes measured are mortality, hospitalization and recurrence of symptoms. For general patient populations, the follow-up periods for mortality ranged from $<24 \mathrm{~h}$ up to $<30$ days, and mortality rates ranged from $0.2-3.5 \%(<24 \mathrm{~h}), 0.3 \%(<48 \mathrm{~h}), 0.3-6.1 \%$ ( $<72 \mathrm{~h}$ ), $0.3 \%-0.7 \%$ (<7 days) up to $1.6 \%$ (<30 days) [26, $34,37,40,43,45,50,52,57]$. The one study reporting on a specific patient population (opioid overdose) reported a $0.8 \%$ mortality rate $<48 \mathrm{~h}$ [44].

The hospitalization follow-up period for general patient populations ranged from $<24 \mathrm{~h}$ up to $<14$ days, and hospitalization rates ranged from $3.3 \%(<24 \mathrm{~h}), \quad 1.0 \%$
(<48 h), 4.5-12.1\% ( $<72 \mathrm{~h}), 5.0-8.1 \%$ ( $<7$ days) up to $5.4-6.2 \%$ (<14 days) [8, 28, 34, 37, 38, 40, 43, 52, 55, 57]. For specific patient populations (hypoglycaemia, people who had fallen, children, people with minor injuries, and people aged $>65$ years) the follow-up periods ranged from $<48 \mathrm{~h}$ up to 12 months, and hospitalization rates ranged from $1.2-7.9 \%(<48 \mathrm{~h}), 2.5-5.1 \%(<72 \mathrm{~h})$ up to $12.0-49.0 \%$ (<12 months) [26, 32, 48, 50, 62, 67, 69, 75].

Recurrence of symptoms for specific patient populations (hypoglycaemia and people who had fallen) varied from $6.1 \%(48 \mathrm{~h}), 7.9 \%(<72 \mathrm{~h}), 4.9 \%$ (<21 days $)$ up to $24.7 \%$ (<6 months) $[32,48,59,76]$.

\section{Existing guidelines, protocols and triage criteria for non- conveyance (Additional file 9: Appendix 8)}

Criteria to guide the (non-) conveyance decision described mostly are abnormal vital functions related to 'breathing' (respiration rate, respiratory distress, dyspnea), abnormal vital functions related to 'circulation' (systolic/diastolic blood pressure, pulse), suspected or confirmed ingestion of alcohol or drugs, and an altered level of consciousness (Glasgow coma scale) [28, 29, 39, $40,43,46,51-54,59,70,72,73,75,79,84,88]$. Ten of these studies described more specific flowcharts, tools, checklist or standard operating procedures for nonconveyance in general $[43,51,72]$, patients who refuse conveyance $[29,40,46]$, and patients who had fallen [84], with supraventricular tachycardia [39], with social problems [28], with hypoglycaemia [53], and post-ictal patients [53].

\section{Professionals competencies and other factors influencing the non-conveyance decision-making process (Table 4)}

Factors influencing the non-conveyance decision-making process are related to the professional, the patient and his relatives, the healthcare process/system, or supportive tools $[26,29,49,51,57,64,65,74,77,78,80,83-89]$ (Table 4). These factors can be present at (a) pre-arrival, when the professional forms an early opinion based on information from the emergency call, during (b) initial patient contact where the ambulance professional gets a first impression of the patient, during (c) patient assessment of vital signs and other parameters, and (d) during the actual (non-) conveyance decision moment [84].

As for professional related factors, two studies described professional competencies needed to perform non-conveyance. These studies showed that additional training for ambulance professionals led to higher nonconveyance rates compared to ambulance professionals who received regular training $[64,77]$. Besides competencies, other professional related factors are weighing of patient risks and personal litigation risk in case of a wrong non-conveyance decision $[87,88]$, experience and intuition of the ambulance professional [89], and 
Table 4 Competences and influencing factors $(n=18)$

\begin{tabular}{|c|c|c|}
\hline Authors (publication year) country [ref] & Competences/influencing factors & Type of factor \\
\hline Alicandro (1995) USA [29] & $\begin{array}{l}\text { The implementation of a (1) high risk card (T1) and (2) online } \\
\text { medical control (T2) for patients with high-risk criteria im } \\
\text { proved the transport rate: T0 2/60 (3.3\%)- T1 7/70 (10.0\%) - T2 } \\
\text { 12/34 (35.3\%) } p=.00003\end{array}$ & $\begin{array}{l}\text { 1. Supportive tools } \\
\text { 2. Healthcare process/system }\end{array}$ \\
\hline Burstein (1998) USA [57] & $\begin{array}{l}\text { The implementation of medical control by telephone to } \\
\text { convince patients who attempt refusal of medical care to be } \\
\text { transported to the ED: } 61 / 130 \text { ( } 47 \%) \text { of the patients was } \\
\text { convinced }\end{array}$ & 1. Healthcare process/system \\
\hline Ebrahimian (2014) Iran [83] & $\begin{array}{l}\text { Affecting factors of EMS staffs' decision about transporting: } \\
\text { 1. patient's condition: } \\
\text { a. Physical health status } \\
\text { b. Socioeconomic status: } \\
\text { i. Patient support system } \\
\text { ii. Patient and his family's educational status } \\
\text { iii. Patient and his family's financial status } \\
\text { c. Cultural background: } \\
\text { i. Confidence } \\
\text { ii. Believes and attitudes } \\
\text { 2. The context of the EMS mission: } \\
\text { a. Characteristics of the mission } \\
\text { b. EMS staffs' characteristics }\end{array}$ & $\begin{array}{l}\text { 1. Patient/relative } \\
\text { 2. Healthcare process/system }\end{array}$ \\
\hline
\end{tabular}

1. Pre-arrival: forming an early opinion from information from the emergency call

2. Initial contact: assessing the need for any immediate action and establishing a report

3. Continuing assessment: gathering and assimilating medical and social information

4. Making a conveyance decision: negotiation, referral and professional defense using professional experience, instinct

Extended care paramedics received additional specialized training in the following "extended care" roles:

1. Geriatric assessments and management

2. End-of-life care

3. Primary wound closure techniques (suturing, tissue adhesive)

4. Point-of-care testing

LTC patients treated by ECPs remained at the LTC facility in 98 of $140(70 \%)$ cases, compared to 21 of $98(21.4 \%)$ of emergency paramedic calls.

Kahalé (2006) Canada [65]

Reasons for non-transport as cited in parent/patient inter views $(n=106)$ :

1. 31/106 (29.2\%) EMS-personnel stated that transport was unnecessary

2. $25 / 106(23.6 \%)$ parents thought that going to the hospital was unnecessary

3. 22/106 (20.8\%) parents wanted to use another method of transportation to seek medical care

4. 5/106 (4.7\%) parents were concerned about costs related to ambulance transports

5. $23 / 106(21.7 \%)$ other

Reasons for not accepting transport (from fieldnotes):

1. Just wanted reassurance, assistance, advice or support/ referral

2. Symptoms had resolved prior to arrival or during assessment

3. Concern over ED waiting time/ED workload

4. Prior negative experience with a hospital

5. Personal reasons: (e.g. 'I just didn't want to go'. 'I was embarrassed by all the fuss'

3. sufficient on-scene treatment

1. Professional

2. Patient/relative 
Table 4 Competences and influencing factors $(n=18)$ (Continued)

\begin{tabular}{|c|c|c|}
\hline & 4.referral to GP & \\
\hline Murphy-Jones (2016) UK [86] & $\begin{array}{l}3 \text { main themes: } \\
\text { 1. Patient wishes (insufficient care plans, nursing care staff } \\
\text { insufficient knowledge of patients' wishes, patients' inability } \\
\text { to express their wishes) } \\
\text { 2. patients' best interest (when patients were not considered } \\
\text { to have the capacity for decision making, paramedics want to } \\
\text { act in their best interest, factors used: diagnosis, } \\
\text { comorbidities, quality of life, wishes and current condition, } \\
\text { risks and benefits of hospitalization, concerns about care } \\
\text { provision in some nursing homes } \\
\text { 3. influence of others (nursing home staff, patients' relatives } \\
\text { and other paramedics) }\end{array}$ & $\begin{array}{l}\text { 1. Patient/relative } \\
\text { 2. Healthcare process/system }\end{array}$ \\
\hline O'Hara (2015) UK [87] & $\begin{array}{l}\text { 7 overarching system influences on decision making: } \\
\text { 1. Increasing demand (of non-emergent cases) } \\
\text { 2. Performance regime and priorities } \\
\text { 3. Access to appropriate care options in case of non- } \\
\text { conveyance to an ED } \\
\text { 4. Disproportionate risk aversion: non-conveyance was } \\
\text { perceived as a risk for both patient and paramedic } \\
\text { 5. Beneficial impact of additional training on decision making } \\
\text { competences } \\
\text { 6. Communication and feedback to crews } \\
\text { 7. Ambulance service resources }\end{array}$ & 1. Healthcare process/system \\
\hline Porter (2007) UK [88] & $\begin{array}{l}\text { Influencing factors: } \\
\text { 1. Patient autonomy } \\
\text { 2. Opinion family/carers } \\
\text { 3. Clinical need as assessed by crew members } \\
\text { 4. Protection of themselves for the risk of litigation by crew } \\
\text { members } \\
\text { 5. Mental capacity of the patient to make a transport decision } \\
\text { 6. Lacking skills or status of the crew member to be judging } \\
\text { the mental capacity of the patient } \\
\text { 7. Back-up of other professionals } \\
\text { 8. Fear of a possible comeback if the non-conveyance } \\
\text { decision turned out to be wrong }\end{array}$ & $\begin{array}{l}\text { 1. Patient/relative } \\
\text { 2. Professional }\end{array}$ \\
\hline Simpson (2014a) Australia [74] & $\begin{array}{l}\text { 6-item predictive model for non-conveyance odds (goodness- } \\
\text { of-fit test indicated good model fit ( } 8 \mathrm{DF}, \times 2=7.43, p=0.49 \text { ), }\end{array}$ & $\begin{array}{l}\text { 1. Patient/relative } \\
\text { 2. Healthcare process/system }\end{array}$ \\
\hline
\end{tabular}
of-fit test indicated good model fit ( $\mathrm{DF}, \mathrm{X} 2=7.43, p=0.49$ ), factors associated with increased odds of a non-conveyance outcome.

1. 65-74 year

2. Lower response priority (90 min response time)

3. The presence of personal alarm

4. The absence of new injury/pain

5. Normal physiology

6. Change in usual level of function post fall

Snooks (2005) UK [89]

Influencing factors on ED conveyance:

1. Experience and intuition of the paramedic

2. Pragmatism: conveyance - the easy option

3. Patient/carer factors

Stark (1990) USA [49]

Predictors for left at Scene Against Medical Advice:

1. Family present $(\beta=-1.87, p=.001)$

2. Disorientation $(\beta=-1.04, p=.04)$

3. Abnormal speech $(\beta=-1.92, p=.05)$

4. Police hold $(\beta=-2.04, p=.03)$

5. Alcohol use $(\beta=1.48, p=.006)$

6. Treated hypoglycemia $(\beta=1.63, p=.05)$

Stuhlmiller (2005) USA [51]

28/137 (20.4\%) patients with whom the online medical control (OLMC) physician spoke during the encounter: $9 / 28$ (32.1\%) agreed to be transported, compared with nine (8.3\%) of the 109 patients who did not speak to the OLMC physician $(p=.001)$

Van der Pols (2011) Netherlands [77]

Motorcycle response vehicles with one ambulance nurse with additional training $(n=468)$ compared to regular ambulance

1. Professional

2. Patient/relative

1. Patient/relative

2. Healthcare process/system

1. Supportive tools

1. Professional 
Table 4 Competences and influencing factors $(n=18)$ (Continued)

$(n=1196)$ : (1) treat and release 129/468 (27.6\%) vs 149/1196 (12.5\%) RR 2.21 (95\%Cl 1.80-2.73)

Vilke (2002) USA [78]

Zorab (1999) UK [80]
Patient reasons $(n=100)$ for patients to refuse transport:

1. 37/100 (37.0\%) did not want transport and ED care

2. $23 / 100(23.0 \%)$ concerned about the cost/coverage of ED

3. $19 / 100$ (19.0\%) paramedics implied no transport was

needed

4. $17 / 100(17.0 \%)$ concerned about the cost of the ambulance

5. 4/100 (4.0\%) language barrier

274/302 (90.7\%) paramedics felt that a lack of health information of the patient had led to a less appropriate carepathway being selected, information that could have helped according to paramedics:

1. Resuscitation status $(n=233,77.2 \%)$

2. Current medication $(n=184,60.9 \%)$

3. Allergy information ( $n=103,34.1 \%)$

4. Previous medical history $(n=262,86.8 \%)$

5. Patient's normal parameters $(n=235,77.8 \%)$

6. End of life care choices $(n=221,73.2 \%)$

7. Information about implanted devices, e.g. pacemakers $(n=106,35.1 \%)$

8.Other, e.g. ECG, mental health records, blood and other test results $(n=38,1.3 \%)$ pragmatism as conveyance being an easy option compared to non-conveyance [89].

For patient related factors, firstly the health status of the patient influenced the non-conveyance decision of the professional $[26,49,65,74,78,83,85,88]$. Only three studies specified these physical conditions: the sufficiency of on-scene treatment [26], if problems/injuries have resolved pre-arrival or were only minor [26, 85], patient physiology [74], the absence of new pain or injury [74], and possible changes in usual level of functioning [74]. A second patient related factor is refusal. Refusal might be related to relatives thinking conveyance is not necessary [65], but also by patients concerns about costs of conveyance or ED care $[65,78]$, or the refusal reasons were not further specified [26, 85]. Thirdly, patient wishes and the patients' best interest are factors that influence a conveyance decision [86].

Influencing factors related to the healthcare system are access/referral to GP or alternative healthcare facility in case of non-conveyance $[26,87]$. To make appropriate conveyance or referral decisions, access to patient information is essential. One study [80] showed that $90.7 \%$ of the ambulance professionals felt that a lack of patient information leads to less appropriate care being selected. To make appropriate decisions, ambulance professionals gave high priority to previous medical history, patient's usual vital signs and resuscitation status as patient information.

Finally, three studies showed that implementing online medical control as supportive tool, where a physician can be contacted by the pre-hospital professional, solely or in combination with a high risk card, increased conveyance rates for patients with high risk criteria or patients who refused conveyance $[29,51,57]$.

\section{Discussion}

This systematic review includes 67 articles that describe non-conveyance in ambulance care from patient safety and ambulance professional perspectives. Our results show that non-conveyance occurs in all types of EMS systems across the world, and that there is a wide variation in non-conveyance rates for general and specific patient populations. These variations might be caused by differences in patient populations (medical acuity and medical necessity to convey), and differences between EMS-systems in terms of triage systems, types of services, educational levels of ambulance professionals, and type of vehicles (conveying and non-conveying) [91-93]. Although non-conveyance in itself is a valid outcome of ambulance care [17], our results do not distinct between justified or unjustified non-conveyance. This can be a focus of future research.

Our review provides a first insight in characteristics of non-conveyed patients. Our results show that patients of all ages and both men and women are represented in the non-conveyance population. Non-conveyed patients most often had a neurological or trauma related complaint or condition. Vulnerable patients as children and elderly, and specific patient groups of people who had fallen or people with hypoglycaemia are relatively high represented in the non-conveyance population. Another subpopulation is patients who refuse care and/or conveyance. From our results it remains unknown what kinds of complaints or conditions these patients have 
from ICD-10 perspective, and what consequences their refusal has from patient-safety perspective.

Although the assessment of vital signs is an important aspect of the primary survey in ambulance care to make appropriate treatment and triage decisions [94], we found only three studies describing vital signs of nonconveyed patients. These studies show that roughly $15 \%$ of the non-conveyed patients have vital signs that deviate from limits. We do not know whether vital signs differ between conveyed and non-conveyed patients. Therefore future research should focus on a comparison of vital signs and follow-up outcomes between conveyed and non-conveyed patient groups. Furthermore, it remains unclear if abnormal vital signs were present in the medical history due to illness or medication use. Poor access to healthcare information systems by ambulance professionals is reported [80], this underlines the possible advantage of access to healthcare information systems in the chain of emergency care, and the accessibility of the general practitioner.

Results show that a significant amount of non-conveyed patient re-enters the (emergency) healthcare system. For instance, $6.1 \%$ of the patients re-enters the EMS-system $<24 \mathrm{~h}$ after non-conveyance, and up to $19.0 \%$ of the patient visits an ED within $48 \mathrm{~h}$ after non-conveyance. From the patient-safety perspective it remains unclear whether these repeated EMS calls and ED visits are based on medical necessity, as it remained unclear in the data which complaints or conditions these patients had during this repeated access to emergency healthcare, and whether it was similar to the initial EMS contact. Furthermore, the studies did not describe whether the re-entry is based on professional referral or self-referral. Clinical practice could benefit from the development of valid quality indicators for patient safety in the chain of emergency care. These could measure systematically (un)justified re-entry of the emergency healthcare system and quality of care provided.

From the professional perspective, our results indicate that the non-conveyance decision-making process is multifactorial, with influences from the professional, the patient and his relatives, the healthcare system, and supportive tools. Our results do not give clear direction which additional competencies ambulance professionals need to make safe non-conveyance decisions, as only two studies describe positive effects of additional training. Studies not included in our review suggest that pre-hospital professionals with additional training on the conveyance decision, and on management of minor illness and injuries, are less likely to convey patients compared to regular ambulance staff $[15,95]$. Initiatives to implement new competencies of pre-hospital professionals in EMS or possibly new professionals with additional competencies in clinical reasoning and conveyance decision-making should be explored and tested regarding patient safety.
As for supportive tools, our results show that there is a limited number of flowcharts, checklists or protocols available to guide non-conveyance decisions for general and specific patient populations. However, it remains unclear how these tools were developed and to what degree they are evidence-based. This urges the need to develop evidence-based supportive tools to guide nonconveyance decision-making for different patient groups. In order to do so, future research should be aimed at identifying factors to guide accurate non-conveyance decision making, to predict non-conveyance in the EMS dispatch phase through tailored triage criteria, or to predict follow-up outcomes such as mortality and re-enters in the emergency healthcare system. This with the aim to support professionals in their decision making and to enhance quality and safety in pre-hospital care.

\section{Limitations of included studies}

As described in the result section, the quality of included studies varied. For the quantitative studies (Additional file 4: Appendix 3, Additional file 5: Appendix 4, Additional file 6: Appendix 5), the quality assessment criteria objective/aim, design, methods of subject/group selection, appropriateness of sample size, description analytical methods, and detailed reporting of results scored good quality. The moderate assessment criteria were description of subject characteristics, outcome definition, and the relationship between results and conclusion. The reporting of estimate of variance was poor, and due to design most studies could not be controlled for confounding. Within the qualitative studies (Additional file 7: Appendix 6) the quality assessment criteria objective/ aim, design, connection to theoretical framework, datacollection and data-analysis scored good quality. The moderate assessment criteria were description of context, sampling strategy, and conclusion supported by results. Use of verification procedures and reflexivity of account were the two poor assessment criteria. Another limitation concerned the studies describing initial complaints and conditions. These studies used different types of classification systems, or systems were lacking. Therefore, we recommend to use one classification system, such as the ICD-10, in future research to enhance generizability and comparability of results.

\section{Study strengths and limitations}

Despite the fact that this systematic review is the most complete and systematic analysis to date of nonconveyance in ambulance care, there are some limitations. A possible limitation is that our review did not cover the entire ambulance care process, as we focused on the phases after ambulance dispatch. Additional research should focus on the accuracy and predictive value of current EMS dispatch systems for non-conveyance 
decisions. Secondly, a meta-analyses was not feasible due to heterogeneity amongst studies. Another limitation concerns the quality assessment tools for quantitative and qualitative designs. A variety of these tools exist without a clear evidence-base. Strengths of our study concern the usage of Cochrane and PRISMA methods and tools to perform and report our research.

\section{Conclusion}

This systematic review shows that non-conveyance occurs in all types of EMS systems across the world, and that a wide variation in non-conveyance rates for general and specific patient populations exists. Patients in the non-conveyance population present themselves with a variety of initial complaints and conditions, although initial complaints or conditions related to trauma and neurology, and vulnerable patients groups such as children, elderly and patients with hypoglycaemia, are well represented. Nevertheless, further insight in characteristics of the non-conveyance population is needed. From patient safety perspective it turns out that a proportion of non-conveyed patients re-enters the emergency healthcare system within one or 2 days after nonconveyance. Why these patients re-enter the emergency healthcare system, and what outcomes these patients have remains unclear. For ambulance professionals the non-conveyance decision-making process is complex and multifactorial, with influences from the professional, the patient and his relatives, the healthcare system (referral or access to general practitioner) and supportive tools. Competencies needed to perform non-conveyance are marginally described, this should be priority in future research. Despite the fact that a limited amount of supportive tools is available for general and specific nonconveyance populations, there is a need to develop evidence-based guidelines and protocols to guide nonconveyance decision-making.

\section{Additional files}

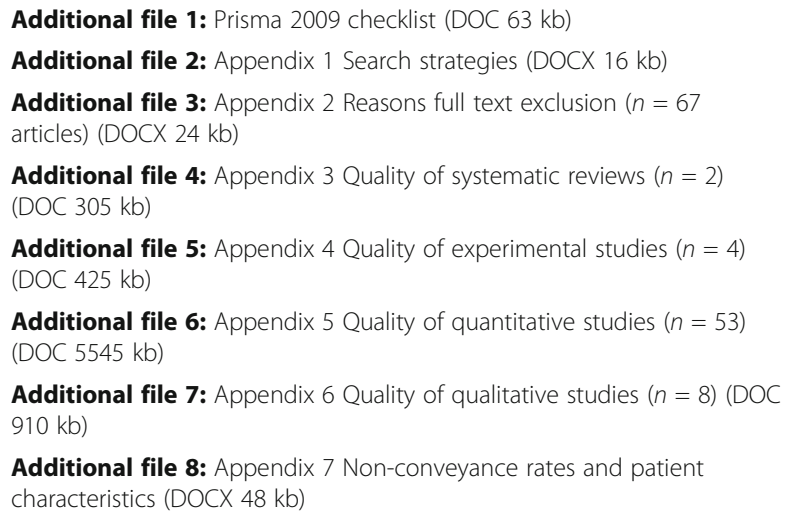

Additional file 9: Appendix 8 Guidelines/protocols/triage criteria (DOCX $29 \mathrm{~kb})$

\section{Acknowledgements}

Not applicable.

\section{Funding}

The study was funded by Dutch National Sector Organization for Ambulance Care, Zwolle, The Netherlands. The funding body had a role in determining the scope and key-questions for this review. There was no role during data collection, selection, analysis and interpretation.

Availability of data and materials

The datasets used and/or analysed during the current study are available from the corresponding author on reasonable request.

\section{Authors' contributions}

Study design (RE, SB, MH, TP, LV). Data collection, selection, extraction and analysis (RE, SB, RS, NT, JL, LV). Quality assessment (RE, SB, RS, NT, JL, LV). Manuscript preparation (RE, SB, RS, NT, JL, MH, TP, LV). All authors read and approved the final manuscript

Ethics approval and consent to participate

Not applicable.

\section{Consent for publication}

Not applicable.

\section{Competing interests}

The authors declare that they have no competing interests.

\section{Publisher's Note}

Springer Nature remains neutral with regard to jurisdictional claims in published maps and institutional affiliations.

\section{Author details}

${ }^{1}$ Research Department of Emergency and Critical Care, HAN University of Applied Sciences, Faculty of Health and Social Studies, PO Box 6960, 6503, GL, Nijmegen, The Netherlands. Radboud University Medical Center, Radboud Institute for Health Sciences, IQ healthcare, Nijmegen, The Netherlands. ${ }^{3}$ Ambulance Service Gelderland-Zuid, Nijmegen, The Netherlands. ${ }^{4}$ Ambulance Service IJsselland, Zwolle, The Netherlands. ${ }^{5}$ Ambulance Academy, Harderwijk, The Netherlands. ${ }^{6}$ Dutch National Sector Organisation for Ambulance Care, Zwolle, The Netherlands. ${ }^{7}$ Radboud University Medical Center, Eastern Regional Emergency Healthcare Network, Nijmegen, The Netherlands.

Received: 21 April 2017 Accepted: 22 June 2017

Published online: 17 July 2017

References

1. Shah MN. The formation of the emergency medical services system. Am J Public Health. 2006;96:414-23.

2. Jensen JL, Bigham BL, Blanchard IE, Dainty KN, Socha D, Carter A, Brown LH, Travers AH, Craig AM, Brown R, Morrison LJ. The Canadian National EMS Research Agenda: a mixed methods consensus study. CJEM. 2013;15:73-82.

3. Lowthian JA, Cameron PA, Stoelwinder JU, Curtis A, Currell A, Cooke MW, McNeil JJ. Increasing utilisation of emergency ambulances. Aust Health Rev. 2011;35:63-9.

4. Richardson LD, Asplin BR, Lowe RA. Emergency department crowding as a health policy issue: past development, future directions. Ann Emerg Med. 2002:40:388-93.

5. Snooks H, Wrigley H, George S, Thomas E, Smith H, Glasper A. Appropriateness of use of emergency ambulances. J Accid Emerg Med. 1998;15:212-5.

6. Anonymous. London ambulance service. In: Managing the conveyance of patients policy and procedures; 2017. http://www.londonambulance.nhs.uk/ talking_with_us/freedom_of_information/classes_of_information/our_ policies_and_procedures.aspx. 
7. Ambulances-in-zicht 2015. Ambulances-in-zicht 2015. 2017: http://www. vrgz.nl/nieuws/ambulances-in-zicht-2015/

8. Cone DC, Kim DT, Davidson SJ. Patient-initiated refusals of prehospital care: ambulance call report documentation, patient outcome, and on-line medical command. Prehosp Disaster Med. 1995;10:3-9.

9. Marks PJ, Daniel TD, Afolabi O, Spiers G, JS NVT. Emergency (999) calls to the ambulance service that do not result in the patient being transported to hospital: An epidemiological study. Emerg Med J. 2002;19:449-52

10. Snooks HA, Dale J, HartleySharpe C, Halter M. On-scene alternatives for emergency ambulance crews attending patients who do not need to travel to the accident and emergency department: A review of the literature. Emerg Med J. 2004;21:212-5.

11. Gratton MC, Ellison SR, Hunt J, Ma OJ. Prospective determination of medical necessity for ambulance transport by paramedics. Prehosp Emerg Care. 2003;7:466-9.

12. Booker MJ, Simmonds RL, Purdy S. Patients who call emergency ambulances for primary care problems: a qualitative study of the decisionmaking process. Emerg Med J. 2014;31:448-52. 5p

13. Booker MJ, Shaw ARG, Purdy S. Why do patients with 'primary care sensitive' problems access ambulance services? A systematic mapping review of the literature. BMJ Open. 2015:5:e007726.

14. Verhage $V$, Tuinstra J, Baller R. Ambulanceritten zonder vervoer van een patiënt. Een verkennende studie naar het ontstaan van eerste hulp geen vervoer ritten. Tijdschr Soc geneeskd. 2014;92:119-24.

15. Tohira H, Williams TA, Jacobs I, Bremner A, Finn J. The impact of new prehospital practitioners on ambulance transportation to the emergency department: a systematic review and meta-analysis. Emerg Med J. 2014;31:e88-94.

16. van de Glind I, Berben S, Zeegers F, Poppen H, Hoogeveen M, Bolt I, van Grunsven $\mathrm{P}$, Vloet L: A national research agenda for pre-hospital emergency medical services in the Netherlands: a Delphi-study. Scand J Trauma Resusc Emerg Med. 2016;24. doi:10.1186/s13049-015-0195-y.

17. Johnson M, O'Hara R, Hirst E, Weyman A, Turner J, Mason S, Quinn T, Shewan J, Siriwardena AN: Multiple triangulation and collaborative research using qualitative methods to explore decision making in pre-hospital emergency care. BMC Med Res Methodol. 2017;17. doi:10.1186/s12874-0170290-z.

18. Leikkola P, Mikkola R, Salminen-Tuomaala M, Paavilainen E. Non-conveyance of patients: challenges to decision-making in emergency are. Clin Nurs Stud. 2016;4:4. https://doi.org/10.5430/cns.v4n4p31

19. Champion HR, Sacco WJ, Gainer PS, Patow SM. The effect of medical direction on trauma triage. JTrauma. 1988;28:235-9.

20. Snooks H, Kearsley N, Dale J, Halter M. New models of care for 999 callers with conditions that are neither life threatening nor serious: results of a national survey. Prehospital Immediate Care. 2000:4:180-2. 3p

21. Higgins JPT, Green S (editors). Cochrane Handbook for Systematic Reviews of Interventions Version 5.0.1 (updated September 2008). The Cochrane Collaboration, 2008. Available from http://training.cochrane.org/handbook

22. Moher D, Liberati A, Tetzlaff J, Altman DG, PRISMA Group. Preferred reporting items for systematic reviews and meta-analyses: the PRISMA statement. Int J Surg. 2010;8:336-41.

23. Shea BJ, Grimshaw JM, Wells GA, Boers M, Andersson N, Hamel C, Porter AC, Tugwell P, Moher D, Bouter LM. Development of AMSTAR: a measurement tool to assess the methodological quality of systematic reviews. BMC Med Res Methodol. 2007;7:10.

24. Kmet LM, Lee RC, Cook LS. Quality assessment criteria for evaluating primary research papers from a varierty of fields. 2004.

25. World Health Organization. The ICD-10 classification of mental and behavioural disorders: clinical descriptions and diagnostic guidelines. World Healht Organization, Geneva.1992, 2017:

26. Mikolaizak AS, Simpson PM, Tiedemann A, Lord SR, Close JC. Systematic review of non-transportation rates and outcomes for older people who have fallen after ambulance service call-out. Australas J Ageing. 2013;32: 147-57.

27. Snooks HA, Carter B, Dale J, Foster T, Humphreys I, Logan PA, Lyons RA, Mason SM, Phillips CJ, Sanchez A, Wani M, Watkins A, Wells BE, Whitfield R, Russell IT. Support and assessment for fall emergency referrals (SAFER 1): Cluster randomised trial of computerised clinical decision support for paramedics. PLosOne. 2014;9(9): e106436. 10.1371/journal.pone.0106436.

28. Snooks H, Kearsley N, Dale J, Halter M, Redhead J, Cheung WY. Towards primary care for non-serious 999 callers: Results of a controlled study of "Treat and Refer" for ambulance crews. Qual Saf Health Care. 2004;13:435-43.
29. Alicandro J, Hollander JE, Henry MC, Sciammarella J, Stapleton E, Gentile D. Impact of interventions for patients refusing emergency medical services transport. Acad Emerg Med. 1995;2:480-5.

30. Key CB, Pepe PE, Persse DE, Calderon D. Can first responders be sent to selected 9-1-1 emergency medical services calls without an ambulance? Acad Emerg Med. 2003;10:339-46.

31. Alrazeeni DM, Sheikh SA, Mobrad A, Al GM, Abdulqader N, Al GM, Al QM, Al Khaldi B. Epidemiology of non-transported emergency medical services calls in Saudi Arabia. Saudi Med J. 2016;37:575-8.

32. Anderson S, Hogskilde PD, Wetterslev J, Bredgaard M, Sorensen MJT, Dahl $J B$, Hogskilde $S$. Appropriateness of leaving emergency medical service treated hypoglycemic patients at home: A retrospective study. Acta Anaesthesiol Scand. 2002:46:464-8.

33. Goldstein J, Jensen JL, Carter AJE, Travers AH, Rockwood K. The epidemiology of prehospital emergency responses for older adults in a provincial EMS system. Can J Emerg Med. 2015;17:491-6.

34. Højfeldt SG, Sørensen LP, Mikkelsen S. Emergency patients receiving anaesthesiologist-based pre-hospital treatment and subsequently released at the scene. Acta Anaesthesiol Scand. 2014;58:1025-31. 7p

35. Kamper M, Mahoney BD, Nelson S, Peterson J. Feasibility of paramedic treatment and referral of minor illnesses and injuries. Prehosp Emerg Care. 2001;5:371-8. 8p

36. Kannikeswaran N, Mahajan PV, Dunne RB, Compton S, Knazik SR. Epidemiology of pediatric transports and non-transports in an urban emergency medical services system. Prehosp Emerg Care. 2007;11:403-7.

37. Knight S, Olson LM, Cook LJ, Mann NC, Corneli HM, Dean JM. Against all advice: an analysis of out-of-hospital refusals of care. Ann Emerg Med. 2003; 42:689-96.

38. Magnusson C, Källenius C, Knutsson S, Herlitz J, Axelsson C. Pre-hospital assessment by a single responder: The Swedish ambulance nurse in a new role: A pilot study. Int Emerg Nurs. 2016;26:32.

39. Minhas R, Vogelaar G, Wang D, Almansoori W, Lang E, Blanchard IE, Lazarenko G, McRae A. A prehospital treat-and-release protocol for supraventricular tachycardia. Can J Emerg Med. 2015;17:395-402.

40. Moss ST, Chan TC, Buchanan J, Dunford JV, Vilke GM. Outcome study of prehospital patients signed out against medical advice by field paramedics. Ann Emerg Med. 1998;31:247-50.

41. Peyravi M, Ortenwal P, Djalali A, KhorramManesh A. An overview of Shiraz emergency medical services, dispatch to treatment. Iran Red Crescent Med J. 2013;15:823-8.

42. Peyravi M, Örtenwall P, Khorram-Manesh A: Can Medical Decision-making at the Scene by EMS Staff Reduce the Number of Unnecessary Ambulance Transportations, but Still Be Safe?. PLOS Currents Disasters. 2015 Jun 30 Edition 1. doi:10.1371/currents.dis.f426e7108516af698c8debf18810aa0a.

43. Pringle RP Jr, Carden DL, Xiao F, Graham DD Jr. Outcomes of patients not transported after calling 911. J Emerg Med. 2005;28:449-54.

44. Rudolph SS, Jehu G, Nielsen SL, Nielsen K, Siersma V, Rasmussen LS. Prehospital treatment of opioid overdose in Copenhagen-Is it safe to discharge on-scene? Resuscitation. 2011;82:1414-8.

45. Schmidt M, Handel D, Lindsell C, Collett L, Gallo P, Locasto D. Evaluating an emergency medical services - Initiated nontransport system. Prehosp Emerg Care. 2006;10:390-3.

46. Selden BS, Schnitzer PG, Nolan FX. Medicolegal documentation of prehospital triage. Ann Emerg Med. 1990;19:547-51.

47. Seltzer AG, Vilke GM, Chan TC, Fisher R, Dunford JV. Outcome study of minors after parental refusal of paramedic transport. Prehosp Emerg Care. 2001;5:278-83.

48. Socransky SJ, Pirrallo RG, Rubin JM. Out-of-hospital treatment of hypoglycemia: refusal of transport and patient outcome. Acad Emerg Med. 1998:5:1080-5.

49. Stark G, Hedges JR, Neely K, Norton R. Patients who initially refuse prehospital evaluation and/or therapy. Am J Emerg Med. 1990;8:509-11.

50. Staudenmayer K, Hsia R, Wang E, Sporer K, Ghilarducci D, Spain D, MacKersie R, Sherck J, Kline R, Newgard C. The forgotten trauma patient: Outcomes for injured patients evaluated by emergency medical services but not transported to the hospital. J Trauma Acute Care Surg. 2012;72:594-600.

51. Stuhlmiller DFE, Cudnik MT, Sundheim SM, Threlkeld MS Jr. CT: Adequacy of online medical command communication and emergency medical services documentation of informed refusals. Acad Emerg Med. 2005;12:970-7. 8p

52. Tohira H, Fatovich D, Williams TA, Bremner AP, Arendts G, Rogers IR, Celenza A, Mountain D, Cameron P, Sprivulis P, Ahern T, Finn J. Is it Appropriate for 
Patients to be Discharged at the Scene by Paramedics? Prehosp Emerg Care. 2016:1-11.

53. Tohira H, Fatovich D, Williams TA, Bremner A, Arendts G, Rogers IR, Celenza A, Mountain D, Cameron P, Sprivulis P, Ahern T, Finn J. Paramedic Checklists do not Accurately Identify Post-ictal or Hypoglycaemic Patients Suitable for Discharge at the Scene. Prehosp Disaster Med. 2016;31:282-93.

54. Vilke GM, Buchanan J, Dunford JV, Chan TC. Are heroin overdose deaths related to patient release after prehospital treatment with naloxone? Prehosp Emerg Care. 1999;3:183-6. 4p

55. Zachariah BS, Bryan D, Pepe PE, Griffin M. Follow-up and outcome of patients who decline or are denied transport by EMS... including commentary by McSwain NE J. Prehosp Disaster Med. 1992;7:359-63. 5p

56. Burstein JL, Henry MC, Alicandro J, Gentile D, Thode HC Jr, Hollander JE. Outcome of patients who refused out-of-hospital medical assistance. Am J Emerg Med. 1996;14:23-6.

57. Burstein JL, Hollander JE, Delagi R, Gold M, Henry MC, Alicandro JM. Refusal of out-of-hospital medical care: Effect of medical-control physician assertiveness on transport rate. Acad Emerg Med. 1998;5:4-8.

58. Cain E, AckroydStolarz S, Alexiadis P, Murray D. Prehospital hypoglycemia: The safety of not transporting treated patients. Prehosp Emerg Care. 2003;7: 458-65.

59. Carter AJ, Keane PS, Dreyer JF. Transport refusal by hypoglycemic patients after on-scene intravenous dextrose. Acad Emerg Med. 2002;9:855-7.

60. Chen JC, Bullard MJ, Liaw SJ. Ambulance use, misuse, and unmet needs in a developing emergency medical services system. Eur J Emerg Med. 1996;3:73-8.

61. Deasy C, Ryan D, O'Donnell C, Cusack S. The impact of a pre-hospital medical response unit on patient care and Emergency Department attendances. Ir Med J. 2008;101:44-6.

62. Haines CJ, Lutes RE, Blaser M, Christopher NC. Paramedic initiated nontransport of pediatric patients. Prehosp Emerg Care. 2006;10:213-9.

63. Hipskind JE, Gren JM, Barr DJ. Patients who refuse transportation by ambulance: a case series. Prehosp disaster Med. 1997;12:278-83.

64. Jensen JL, Travers AH, Bardua DJ, Dobson T, Cox B, McVey J, Cain E, Merchant R, Carter AJE. Transport outcomes and dispatch determinants in a paramedic long-term care program: a pilot study. CAN J EMERG MED. 2013; $15: 206-13.8 p$

65. Kahale J, Osmond MH, Nesbitt L, Stiell IG. What are the characteristics and outcomes of nontransported pediatric patients? Prehosp Emerg Care. 2006; 10:28-34.

66. Lerner EB, Billittier AJ 4th, Lance DR, Janicke DM, Teuscher JA. Can paramedics safely treat and discharge hypoglycemic patients in the field? Am J Emerg Med. 2003;21:115-20.

67. Mechem CC, Kreshak AA, Barger J, Shofer FS. The short-term outcome of hypoglycemic diabetic patients who refuse ambulance transport after outof-hospital therapy. Acad Emerg Med. 1998;5:768.

68. Newton PR, Naidoo R, Brysiewicz P. The appropriateness of emergency medical service responses in the eThekwini district of KwaZulu-Natal, South Africa. S Afr Med J. 2015;105:844.

69. Persse $D E$, Key CB, Baldwin JB. The effect of a quality improvement feedback loop on paramedic-initiated nontransport of elderly patients. Prehosp Emerg Care. 2002;6:31-5

70. Schmidt TA, Atcheson R, Federiuk C, Mann NC, Pinney T, Fuller D, Colbry K. Hospital follow-up of patients categorized as not needing an ambulance using a set of emergency medical technician protocols. Prehosp Emerg Care. 2001;5:366-70.

71. Schmidt TA, Mann NC, Federiuk CS, Atcheson RR, Fuller D, Christie MJ. Do patients refusing transport remember descriptions of risks after initial advanced life support assessment? Acad Emerg Med. 1998;5:796-801.

72. Schmidt T, Atcheson R, Federiuk C, Mann NC, Pinney T, Fuller D, Colbry K. Evaluation of protocols allowing emergency medical technicians to determine need for treatment and transport. Acad Emerg Med. 2000;7:663-9.

73. Simpson PM, Bendall JC, Tiedemann A, Lord SR, Close JC. Epidemiology of emergency medical service responses to older people who have fallen: a prospective cohort study. Prehosp Emerg Care. 2014;18:185-94.

74. Simpson PM, Bendall JC, Toson B, Tiedemann A, Lord SR, Close JC. Predictors of nontransport of older people who have fallen who receive ambulance care. Prehosp Emerg Care. 2014;18:342-9.

75. Strote J, Simons R, Eisenberg M. Emergency medical technician treatment of hypoglycemia without transport. Am J Emerg Med. 2008;26:291-5.

76. Tiedemann A, Mikolaizak AS, Sherrington C, Segin K, Lord SR, Close JC Older people who have fallen attended to by an ambulance but not transported to hospital: a vulnerable population at high risk of future falls. Aust N Z J Public Health. 2013;37:179-85.

77. Van Der Pols H, Mencl F, De Vos R. The impact of an emergency motorcycle response vehicle on prehospital care in an urban area. Eur J Emerg Med. 2011;18:328-33.

78. Vilke GM, Sardar W, Fisher R, Dunford JD, Chan TC. Follow-up of elderly patients who refuse transport after accessing 9-1-1. PrehospEmergCare. 2002:6:391-5.

79. Gerlacher GR, Sirbaugh PE. Macias CG: Prehospital evaluation of nontransported pediatric patients by a large emergency medical services system. Pediatr Emerg Care. 2001;17:421-4.

80. Zorab O, Robinson M, Endacott R. Are prehospital treatment or conveyance decisions affected by an ambulance crew's ability to access a patient's health information? BMC Emerg Med. 2015;15:1-7. 7p

81. Shaw D, Dyas JV, Middlemass J, Spaight A, Briggs M, Christopher S, Siriwardena AN. Are they really refusing to travel? A qualitative study of prehospital records. BMC Emergency Med. 2006;6. doi:10.1186/1471-227X-68.

82. Burrell L, Noble A, Ridsdale L. Decision-making by ambulance clinicians in London when managing patients with epilepsy: a qualitative study. Emerg Med J. 2013;30:236-40. 5p

83. Ebrahimian $\mathrm{A}$, Seyedin $\mathrm{H}$, JamshidiOrak R, Masoumi G. Exploring factors affecting emergency medical services staffs' decision about transporting medical patients to medical facilities. Emerg Med Int. 2014. doi:10.1155/ 2014/215329.

84. Halter M, Vernon S, Snooks H, Porter A, Close J, Moore F, Porsz S. Complexity of the decision-making process of ambulance staff for assessment and referral of older people who have fallen: a qualitative study. Emerg Med J. 2011;28:44-50. 7p

85. Keene T, Davis M, Brook C. Characteristics and outcomes of patients assessed by paramedics and not transported to hospital: A pilot study. Australas J Paramedicine. 2015;12:1-7.

86. Murphy-Jones G, Timmons S. Paramedics' experiences of end-of-life care decision making with regard to nursing home residents: an exploration of influential issues and factors. Emerg Med J: EMJ. 2016;0:1-5. doi:10.1136/ emermed-2015-205405.

87. O'Hara R, Johnson M, Siriwardena AN, Weyman A, Turner J, Shaw D, Mortimer P, Newman C, Hirst E, Storey M, Mason S, Quinn T, Shewan J. A qualitative study of systemic influences on paramedic decision making: care transitions and patient safety. J Health Serv Res Policy. 2015;20:45-53.

88. Porter A, Snooks H, Youren A, Gaze S, Whitfield R, Rapport F, Woollard M. 'Should I stay or should I go?' Deciding whether to go to hospital after a 999 call. J Health Serv Res Policy. 2007;12:51:32-8.

89. Snooks HA, Kearsley N, Dale J, Halter M, Redhead J, Foster J. Gaps between policy, protocols and practice: A qualitative study of the views and practice of emergency ambulance staff concerning the care of patients with nonurgent needs. Qual Saf Health Care. 2005;14:251-7.

90. Hiälte L, Suserud B, Herlitz J, Karlberg I. Why are people without medical needs transported by ambulance? A study of indications for pre-hospital care. Eur J Emerg Med. 2007;14:151.

91. Billittier AJ, Lerner EB, Moscati RM, Young G. Triage, transportation, and destination decisions by out-of-hospital emergency care providers. Prehosp Disaster Med. 1998;13:22-7.

92. Eric Carlström, Lars Fredén, The first single responders in Sweden Evaluation of a pre-hospital single staffed unit. Int Emerg Nurs, Volume 32, May 2017, Pages 15-19, ISSN 1755-599X, https://doi.org/10.1016/j.ienj.2016. 05.003. http://www.sciencedirect.com/science/article/pii/ S1755599X16300490).

93. Hoyle S, Swain AH, Fake P, Larsen PD. Introduction of an extended care paramedic model in New Zealand. Emerg Med Australas. 2012;24:652-6.

94. Coats TJ, Wilson AW, Cross FW. On-scene medical decision making and overtriage. Br J Surg. 1993;80:1291.

95. Bloemhoff A, Schoonhoven L, de Kreek AJ, van Grunsven PM, Laurant MG, Berben SA. Solo emergency care by a physician assistant versus an ambulance nurse: a cross-sectional document study. Scand J Trauma Resusc Emerg Med. 2016;24. doi:10.1186/s13049-016-0279-3. 